\title{
MAGNETIC PROPERTIES OF A KRAMERS DOUBLET. AN UNIVOCAL BRIDGE BETWEEN EXPERIMENTAL RESULTS AND THEORETICAL PREDICTIONS
}

\author{
P. J. Alonso and J. I. Martínez
}

Instituto de Ciencia de Materiales de Aragón (Universidad de Zaragoza-Consejo Superior de Investigaciones Científicas) Facultad de Ciencias, Universidad de Zaragoza, C/ Pedro Cerbuna 12, 50009 Zaragoza (Spain)

\begin{abstract}
The magnetic response of a Kramers doublet is analysed in a general case taking into account only the formal properties derived from time reversal operation. It leads to a definition of a matrix $G$ (gyromagnetic matrix) whose expression depends on the chosen reference frame and on the Kramers conjugate basis used to describe the physical system. It is shown that there exists a reference frame and a suitable Kramers conjugate basis that gives a diagonal form for the $G$-matrix with all non-null elements having the same sign. A detailed procedure for obtaining this canonical expression of $G$ is presented when the electronic structure of the KD is known regardless the level of the used theory. This procedure provides a univocal way to compare the theoretical predictions with the experimental results obtained from a complete set of magnetic experiments. In this way the problems arising from ambiguities in the $g$-tensor definition are overcome. This procedure is extended to find a spinHamiltonian suitable for describing the magnetic behaviour of a pair of weakly coupled Kramers systems in the multispin scheme when the interaction between the two moieties as well as the individual Zeeman interaction are small enough as compared with ligand field splitting. Explicit relations between the physical interaction and the parameters of such a spinHamiltonian are also obtained.
\end{abstract}

KEYWORDS: Kramer doublet, spin-Hamiltonian, $g$-tensor, exchange coupled pair 


\section{INTRODUCTION}

After its introduction by Pryce [PR50] the spin Hamiltonian (SH) has been commonly used as a powerful tool for describing electron paramagnetic resonance (EPR) results. The SH was initially applied to the study of the paramagnetic behaviour of $3 \mathrm{~d}$ ions with an orbitally non-degenerate ground state. Shortly after, it was extended by Elliott and Stevens [ES51, ES52, ES53, EL53] for describing the paramagnetic properties of $4 f$ ions. The introduction of the effective Hamiltonian concept by Soliverez [SO69] as an alternative way to time independent perturbation techniques gave a formal support to the Pryce SH derivation and a formal derivation of the $\mathrm{SH}$ in a general case was given in [CA72, DU93]. In the early construction by Pryce [PR50] and by Abragam and Pryce [AP51] the physical meaning of the different terms in the SH was obvious since the spin of the paramagnetic entity is a good quantum number. The $\mathrm{SH}$ is written in term of an effective spin that coincide with the physical spin of the ground state orbital singlet. However, this simple interpretation is lost in a general situation where the $\mathrm{SH}$ is a class of effective Hamiltonian written in term of spin operators acting on the $\left|S^{\prime}, M^{\prime}\right\rangle$ states of a fictitious spin, $S^{\prime}$, which is suitable to describe the behaviour of a set of $n$ states of the actual physical system, well separated in energy from the excited states. $S^{\prime}$ is determined by $2 S^{\prime}+1=n$ [RM01]. This situation appears in the SH introduced by Elliot and Stevens [ES53, El53] which is written in term of an effective spin determined by the degeneration of the low energy crystal field level of a rare-earth. A clear discussion about the effective spin and fictitious spin concepts has been recently published by Rudovicz et al. [RU87, RK15b]. The connection between the physical Hamiltonian, which accounts for the physical interactions that determine the behaviour of the paramagnetic entity, and the SH written in term of an effective spin and useful for describing the experimental findings is a central question. [RK15b]

All these issues were already underlined by Low [LO60] who wrote "The spinHamiltonian is used to give a shorthand description of the experimental results. The experimentalist determines the parameters in the spin Hamiltonian, $g$ factors, initial splittings and hyperfine constants". Below this, the author wrote "One must then find a model of crystals field which corresponds to the spin Hamiltonian and which will explain the observed parameters”, giving an added value to the $\mathrm{SH}$ as a bridge between the experimental results and the microscopical description of the paramagnetic entity. As it was recently pointed out by Neese [NE11], this point of view is still a central concept for the interpretation of the SH parameters. The computing codes today are more powerful in calculating electronic properties 
of paramagnetic entities [BO03, KB04, BO06, NE07, NE11, SN12, MG13, SK13, GN13] and the experimental techniques have become more sophisticated allowing to obtain more wide and precise information about $\mathrm{SH}$ parameters [SJ01]. However, the existence of some ambiguities in the definition of the SH parameters, which was already noted in the early times [GR67, FO67, GM75, RS76, RO88] as well as in recent works [BO06, GN13], is a hurdle for the direct comparison between the experimentally derived parameters and the calculated one.

For instance, the SH description of a Kramers doublet $(n=2)$ is done by introducing a fictitious spin $S^{\prime}=1 / 2$. Forgetting any other interaction, this $S H$ only contains a linear term in the magnetic field, which accounts for the Zeeman interaction and is characterized by a $g$ "tensor" [AB70a] although its tensor behaviour is unclear and some authors prefer to use the $g$-“matrix" term [BO06]. . In this case (a KD) the ambiguities that appear in the SH definition are the signs of individual principal values of such a $g$-“tensor”. However, the sign of its determinant, which coincide with the product of the three principal $g$-values, can be determined by a suitable experiment that makes use of circular polarised microwave [AB70b, PO83]. This sign has a physical meaning because it gives the precession direction of the magnetic moment under a magnetic field [PR59, AB70b]. However, most of the spectrometer has not a circular polarised microwave source and the measured spectra only provide the absolute value of the principal $g$-values [PO83].

Some examples of Kramers ions whose magnetic properties are currently described by using the former approximation are $d^{5}$-ions in the so called low spin (LS) configuration, specially LS-Fe(III) [BB56, GR57, TA77, BO77, RI94, GA98a, GA98b, WA99, BS03, WA04, AM07, ZB09], LS- $d^{7}$, mainly LS-Co(II) [GR58, BM70, BC73, GA75, NK78, AA09, GM14] and rare earth-ions with an odd number of $4 f$ electrons.

In this context several works look for criteria to find a non-ambiguous calculation of the sign of the principal values of the $g$-tensor. For instance, a recent discussion about the uniqueness of the $g$-tensor definition of a Kramers doublet has been presented by Chibotaru et al. [CC08, CU12] following a similar approach to that given earlier by McGarvey [GA98b]. It was postulated that the $g$-tensor should reflect the symmetry of the physical system when this symmetry is high enough and it was assumed that the $g$-tensor in a general case should show a continuous dependence on any deformation that transforms the actual geometry to a suitable high symmetry. Other attempts by Bolvin [BO06] part from the definition of the $g$-"matrix" given in [GM75]. The author imposes that all principal values should be positive and so, the experimental possibility of detecting systems with a negative product of the principal values, 
as actually was reported for a neptunyl ion [PR59], is forgotten. On the other hand, other authors refused to calculate these signs as they cannot be experimentally detected. This negative position appeared in the seminal work by Gerloch and McMeeking [GM75], where a general procedure for calculating the " $g$-tensor" of a paramagnetic entity without any predetermined symmetry condition was given, as well as in recent papers where more sophisticated techniques were employed to calculate the " $g$-tensor" [GN13].

Here we address the question by using a different approach. The main goal is to determine which magnitudes can be calculated when a magnetic field is applied and which of them are independent of the KD representation (the basis used to describe it). These quantities will be the only ones that can be measured (observables). This task is undertaken in a general case assuming that a basis of wave-functions spanning the KD is known without any previous condition; we will only take into account the transformation properties of the wave-functions under time reversal, which are consequence of the Kramers nature of the system. Consequently the results will be independent on the theoretical level used for calculating those wave-functions.

After a brief review of the formal properties of a KD (section 2) our description is presented in section 3. Our formalism allows building a fictitious spin Hamiltonian defined by a true $g$-tensor suitable for describing the magnetic behaviour of the system and precise rules are given for a straightforward comparison between the experimental results and the calculated values. This formulation does not depend on a particular symmetry of the physical system. However, the $g$-tensor (calculated or experimentally determined) should reflect the symmetry of the actual physical system (section 4). With the aim of illustrating our results an hypothetical case is thoroughly developed in section 5 and some selected example taken from the literature are discussed in section 6.

On the other hand, the study of molecular structures having a number of interacting paramagnetic entities is a field of great interest nowadays. A hot topic is the SH description of molecular magnets using either the giant spin approach or the multispin one [MG10a, MG10b, MP10, MG13, MC14, RM14, RK15b]. In this context, a search for the description of a coupled pair of paramagnetic ions is a useful stating point [AB70c, OH72, KA85, CA86, BG90, KA93, BO99]. In the case of weakly interacting Kramers moieties, e. g. a pair of rare earth ions with an odd number of $4 f$ electrons, a desired goal will be to obtain an effective Hamiltonian acting within the low-lying Kramers doublets and to relate the phenomenological parameters of such a SH with more fundamental interactions [PT03. PT10, PT11]. In the last 
section of this paper we sketch how our results can be extended to find a SH suitable for describing the magnetic behaviour of a pair of weakly interacting Kramers systems at temperature low enough for only the lowest energy levels were populated. The application of the method is illustrated by means a hypothetical example.

\section{FORMAL PROPERTIES OF A KRAMERS DOUBLET}

Let us recall briefly some formal properties of a Kramers doublet (KD), based on the detailed discussion in [AB70a]. In the following we will denote the time reversal operator as $T$, which for a Kramers systems obeys $T^{2}=-I$, where $I$ is the identity.

i) For any state, $\Phi$, in a $\mathrm{KD}, T \Phi$ is orthogonal to $\Phi$. Consequently, $\Phi$ and $T \Phi$ are linearly independent and then $\{\Phi, T \Phi\}$ is a basis of the KD. It forms a pair of Kramer conjugate states, whereas the basis $\{\Phi, T \Phi\}$ is a Kramers conjugate basis (KCB). If $\left\{\Phi_{1}\right.$, $\left.T \Phi_{1}\right\}$ and $\left\{\Phi_{2}, T \Phi_{2}\right\}$ are two KCB, the unitary transformation $U$ relating them belongs to the $\mathbf{S U}(2)$ group. Reciprocally, any unitary operation of the $\mathbf{S U}(2)$ group transforms any KCB to another KCB.

ii) A linear operator, $O$, is time-even (time-odd) if $\mathrm{TOT}^{-1}=\mathrm{O}^{+}\left(\mathrm{TOT}^{-1}=-\mathrm{O}^{+}\right)$, where $\mathrm{O}^{+}$denotes the adjoint operator of $O$. In particular, if $O$ is an observable (Hermitian or selfadjoint) then it is time-even (time-odd) if $\mathrm{TOT}^{-1}=O\left(\mathrm{TOT}^{-1}=-\mathrm{O}\right)$. Note that if $O_{1}$ and $O_{2}$ are two operators having a definite time parity, the product, $\mathrm{O}_{1} \mathrm{O}_{2}$, has no definite time parity unless $O_{1}$ and $O_{2}$ commute or anticommute. Given a pair of Kramers conjugate states, if $O$ is a time-even operator then $\langle\Phi|O| \Phi\rangle=\langle T \Phi|O| T \Phi\rangle$ and $\langle\Phi|O| T \Phi\rangle=0$. On the other hand, if $O$ is a time-odd operator then $\langle\Phi|O| \Phi\rangle=-\langle T \Phi|O| T \Phi\rangle$.

iii) Let us now consider an observable, $O$, in a $\mathrm{KD}$. $O$ is time-even if and only if $O$ is a scalar operator (a multiple of the identity) and $O$ is time-odd if and only if $O$ is a traceless operator (for definition of trace of an operator see [ME64c]). Moreover, any observable, $O$, can be decomposed as a sum of a time-even observable and a time-odd observable and this decomposition is unique. Then, the real linear space of the observables in a $\mathrm{KD}$ is a direct sum of the real subspaces of time-even (scalar) and time-odd (traceless) observables.

\section{A KRAMERS DOUBLET UNDER AN APPLIED MAGNETIC FIELD}


Let be an electronic system in the presence of a magnetic field $\vec{B}$. Its intensity will be denoted by $B$ and its direction is given by the unitary vector $\hat{u}$ where $\vec{B}=B \hat{u}$. If the total orbital and spin angular momenta are $\vec{L}$ and $\vec{S}$, respectively, the Zeeman contribution to the Hamiltonian is given by:

$$
H_{Z e}=\mu_{B} \vec{B} \cdot\left(\vec{L}+g_{e} \vec{S}\right)=\mu_{B} \vec{B} \cdot \vec{N}
$$

where $\mu_{\mathrm{B}}$ and $g_{e}$ indicate the Bohr magneton and the free electron gyromagnetic factor, respectively, and the vector operator under $\mathbf{S O ( 3 ) , ~} \vec{N}=\vec{L}+g_{e} \vec{S}$, has been introduced. Since $\vec{L}$ and $\vec{S}$ are time-odd operator then $\vec{N}$ is likewise.

Given a reference frame, $\varsigma \equiv(x, y, z),(1)$ is written as:

$$
H_{z e}=\mu_{B}\left(B_{x} N_{x}+B_{y} N_{y}+B_{z} N_{z}\right)
$$

where $\left(B_{x}, B_{y}, B_{z}\right)$ are the components of $\vec{B}$ and $\left(N_{x}, N_{y}, N_{z}\right)$ the components of the vector operator $\vec{N}$, which are also time-odd traceless Hermitian operators. By introducing the column matrix $u$, whose elements are given by the components of $\hat{u}$ in the reference frame $\varsigma \equiv$ $(x, y, z),(2)$ is expressed as:

$$
H_{z e}=\mu_{B} B\left(u_{x} N_{x}+u_{y} N_{y}+u_{z} N_{z}\right)=\mu_{B} B\left(u^{t} \cdot N\right)
$$

where $N$ is a column matrix, whose elements are given by the components of the vector operator $\vec{N}$ in the chosen reference frame.

Let us consider now a $\mathrm{KD}$. When a particular $\mathrm{KCB},\{\Phi, T \Phi\}$, is chosen, the matrix expression of each one of the $\vec{N}$ components will take the form:

$$
N_{k, \Phi}=\frac{1}{2}\left(\begin{array}{cc}
G_{k z} & G_{k x}-i G_{k y} \\
G_{k x}+i G_{k y} & -G_{k z}
\end{array}\right) \quad(k=x, y, z)
$$

where the $1 / 2$ factor is introduced for convenience and the $G_{k, j}(k, j=x, y, z)$ are real numbers. In this way, given a particular reference frame, $\varsigma \equiv(x, y, z)$, and a $\mathrm{KCB},\{\Phi, T \Phi\}$, a $G_{\zeta, \Phi}$ matrix is built as:

$$
G_{\zeta, \Phi}=\left(\begin{array}{lll}
G_{x x} & G_{x y} & G_{x z} \\
G_{y x} & G_{y y} & G_{y z} \\
G_{z x} & G_{z y} & G_{z z}
\end{array}\right)
$$


It is important to note that as it depends on the chosen $\mathrm{KCB}$, the $G_{\zeta, \Phi}$ matrix is not, in principle, observable. The question now is to know how the $G_{\zeta, \Phi}$ matrix transforms when another reference frame or a different KCB are used.

Firstly, let us fix the KCB and consider any other reference frame, $\varsigma^{\prime} \equiv\left(x^{\prime}, y^{\prime}, z^{\prime}\right)$, related with the previous one by a direct rotation $R \in \mathbf{S O}(3)$.

$$
\left(\begin{array}{l}
x^{\prime} \\
y^{\prime} \\
z^{\prime}
\end{array}\right)=R \cdot\left(\begin{array}{l}
x \\
y \\
z
\end{array}\right)
$$

Then, since $\vec{N}$ is a vector operator under the $\mathbf{S O}(3)$ group, its components in both, $\varsigma$ and $\varsigma^{\prime}$, reference frame are related by

$$
\left(\begin{array}{l}
N_{x^{\prime}} \\
N_{y^{\prime}} \\
N_{z^{\prime}}
\end{array}\right)=R \cdot\left(\begin{array}{l}
N_{x} \\
N_{y} \\
N_{z}
\end{array}\right)
$$

and, consequently, for the fixed KCB, $\{\Phi, T \Phi\}$,

$$
G_{\varsigma^{\prime}, \Phi}=R \cdot G_{\zeta, \Phi}
$$

On the other hand, given a reference frame, $\varsigma \equiv(x, y, z)$, if $\left\{\Phi^{\prime}, T \Phi^{\prime}\right\}$ is another KCB, which is related with the previous one by:

$$
\left(\begin{array}{c}
\Phi^{\prime} \\
T \Phi^{\prime}
\end{array}\right)=U^{*} \cdot\left(\begin{array}{c}
\Phi \\
T \Phi
\end{array}\right)
$$

with $U \in \mathbf{S U}(2)$, then:

$$
G_{\zeta, \Phi^{\prime}}=G_{\zeta, \Phi} \cdot \omega(U)^{t}=G_{\zeta, \Phi} \cdot \omega\left(U^{+}\right)
$$

where $\omega$ stands for the homomorphism from SU(2) onto SO(3) [TI64].

Consequently, if both, the reference frame and the KCB are changed according to (6) and (9), respectively, then

$$
G_{\zeta^{\prime}, \Phi^{\prime}}=R \cdot G_{\zeta, \Phi} \cdot \omega(U)^{t}=R \cdot G_{\zeta, \Phi} \cdot \omega\left(U^{+}\right)
$$

As both, $R$ and $\omega\left(U^{+}\right)$, matrices belong to the $\mathbf{S O}(3)$ group their determinants are equal

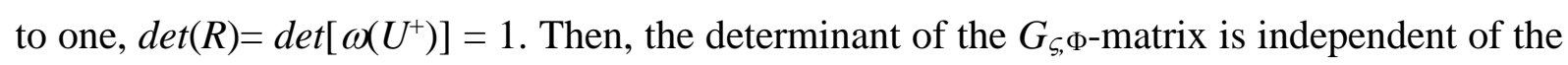
$\mathrm{KCB}$ and the reference frame used. This common value will be denoted by $\Delta$ : 


$$
\Delta=\operatorname{det}\left(G_{\zeta^{\prime}, \Phi^{\prime}}\right)=\operatorname{det}\left(G_{\zeta, \Phi}\right)
$$

Consequently, $\Delta$ is a property of the KD; its sign will be designated by s. $s=\Delta /|\Delta|$ if $\Delta \neq 0$ and, as a convention, we will take $s=+1$ if $\Delta=0$.

A noticeable consequence of the relation (11) is the existence of some reference frame, $\Sigma \equiv(X, Y, Z)$, and some $\mathrm{KCB},\{\Psi$, T $\Psi\}$, such that the $G_{\Sigma, \Psi}$ matrix is diagonal.

Let us consider an arbitrary reference frame, $\varsigma \equiv(x, y, z)$, and an arbitrary $\mathrm{KCB},\{\Phi$, $T \Phi\}$, to build the $G_{\varsigma, \Phi}$ matrix. Taking into account the singular value decomposition of a matrix (see appendix A for details) there exist two matrices $P, Q \in \mathbf{S O}(3)$ and a diagonal matrix $G_{d}=\operatorname{diag}\left(G_{X}, G_{Y}, G_{Z}\right)$ that verify $G_{\zeta, \Phi}=P \cdot G_{d} \cdot Q^{t}$, where $Q^{t}$ is the transpose of $Q$. Equivalently:

$$
P^{t} \cdot G_{\zeta, \Phi} \cdot Q=G_{d}
$$

The number of non-null diagonal elements in $G_{d}$ coincides with the rank of the $G_{\zeta, \Phi}$ matrix; consequently, it does not depend on the reference frame and on the KCB used. The common value of this rank will be denoted hereafter by $r$. Besides, it can be assumed that $r>$ 0 . If $r=0$ then $G_{\zeta, \Phi}=0$ and no magnetic response would take place.

As $P \in \mathbf{S O}(3)$ a new reference frame, $\Sigma \equiv(X, Y, Z)$, can defined from the initial one, $\varsigma$ $\equiv(x, y, z)$, by:

$$
\left(\begin{array}{l}
X \\
Y \\
Z
\end{array}\right)=P^{t} \cdot\left(\begin{array}{l}
x \\
y \\
z
\end{array}\right)
$$

and, because $\omega$ is a homomorphism from $\mathbf{S U}(2)$ onto $\mathbf{S O}(3)$, it is possible to find a matrix $U_{0}$ $\in \mathbf{S U}(2)$ such as $\omega\left(U_{0}\right)=Q^{t}$. Then, a new $\mathrm{KCB},\{\Psi, \mathrm{T} \Psi\}$, can be introduced by:

$$
\left(\begin{array}{c}
\Psi \\
T \Psi
\end{array}\right)=U_{0}^{*} \cdot\left(\begin{array}{c}
\Phi \\
T \Phi
\end{array}\right)
$$

According to (11) $G_{\Sigma, \Psi}$ is given by:

$$
G_{\Sigma, \Psi}=P^{t} \cdot G_{\zeta, \Phi} \cdot \omega\left(U_{o}\right)^{t}=P^{t} \cdot G_{\zeta, \Phi} \cdot Q=G_{d}
$$

The question now is how to obtain a reference frame and a KCB that give the diagonal expression of $G_{\Sigma, \Psi}(16)$ 
Firstly, note that from (13) it follows that

$$
G_{\zeta, \Phi} \cdot G_{\zeta, \Phi}^{t}=\left(P \cdot G_{d} \cdot Q^{t}\right) \cdot\left(P \cdot G_{d} \cdot Q^{t}\right)^{t}=P \cdot G_{d}^{2} \cdot P^{t}
$$

Then, $G_{\zeta, \Phi} \cdot G_{\zeta, \Phi}^{t}$ is a positive-semidefinite symmetric matrix, which does not depend on the chosen KCB. It only depends on the reference frame we use. To stress this point it will be designated as $\Gamma_{\varsigma}=G_{\zeta, \Phi} \cdot G_{\zeta, \Phi}^{t}$. Note that $\Gamma_{\Sigma}=G_{d}^{2}$ is diagonal and its eigenvalues, which are non-negative, do not depend on the used KCB either. Their positive square roots give the $\left|G_{r}\right|$ $(r=X, Y, Z)$ values. It is important to note that $G_{X} G_{Y} G_{Z}=\operatorname{det}\left(G_{\Sigma, \Psi}\right)=\operatorname{det}\left(G_{\zeta, \Phi}\right)=\Delta$, and this value is independent of the KCB used. Since the sign of $\operatorname{det}\left(G_{\Sigma}\right)$, s, is determined, see above, only four sets for $G_{r}(r=X, Y, Z)$ values can be, in principle, derived: $\left(s\left|G_{X}\right|, s\left|G_{Y}\right|, s\left|G_{Z}\right|\right)$, $\left(s\left|G_{X}\right|,-s\left|G_{Y}\right|,-s\left|G_{Z}\right|\right),\left(-s\left|G_{X}\right|, s\left|G_{Y}\right|,-s\left|G_{Z}\right|\right)$ and $\left(-s\left|G_{X}\right|,-s\left|G_{Y}\right|, s\left|G_{Z}\right|\right)$.

Rewriting (17) as $\Gamma_{\varsigma} P=P \Gamma_{\Sigma}$, it is easy to see that the columns of $P$ correspond to the coordinates of the principal directions of $\Gamma_{\varsigma}=G_{\zeta, \Phi} \cdot G_{\zeta, \Phi}^{t}$ in the $\varsigma \equiv(x, y, z)$ reference frame. These principal directions coincide with the axes of the $\Sigma \equiv(X, Y, Z)$ reference frame, which, consequently, does not depend on the chosen KCB. However, apart from the existence of degeneracies (see below, section 4), there are some ambiguities in the choice of the $\Sigma \equiv(X, Y$, $Z$ ) reference frame, which involve $\pi$ rotations around any of the $X, Y$ or $Z$ axes; we can use instead of $\Sigma \equiv(X, Y, Z)$ any of the $\Sigma^{X} \equiv(X,-Y,-Z), \Sigma^{Y} \equiv(-X, Y,-Z)$ or $\Sigma^{Z} \equiv(-X,-Y, Z)$ reference frames. That is equivalent to a substitution of $G_{\Sigma, \Psi}=\operatorname{diag}\left(G_{X}, G_{Y}, G_{Z}\right)$ in (16) by $G_{\Sigma^{\mathrm{x}}, \Psi}=$ $\operatorname{diag}\left(G_{X},-G_{Y},-G_{Z}\right), G_{\Sigma^{\mathrm{Y}}, \Psi}=\operatorname{diag}\left(-G_{X}, G_{Y},-G_{Z}\right)$ or $G_{\Sigma^{\mathrm{Z}}, \Psi}=\operatorname{diag}\left(-G_{X},-G_{Y}, G_{Z}\right)$, respectively.

The election of the ordering of the axes is an additional ambiguity. To overcome this, the condition $\left|G_{X}\right| \leq\left|G_{Y}\right| \leq\left|G_{Z}\right|$ could be imposed. Since $\operatorname{rank}\left(G_{\Sigma, \Psi}\right)=r>0$, see above, this implies that $G_{Z} \neq 0$. This is an arbitrary choice that will be useful for the following discussion. However, other choices could be as good as this one and they are preferred in some cases to highlight symmetry aspects (see below).

After determining a reference frame, $\Sigma \equiv(X, Y, Z)$, that makes $\Gamma_{\varsigma}$ diagonal we will show how a $\operatorname{KCB}\{\Psi, T \Psi\}$ giving a diagonal expression for $G_{\Sigma, \Psi}$ can be obtained. The existence of such a basis is guaranteed by (16). Moreover, we will see that if $\{\Psi, T \Psi\}$ is any of those KCB, the pairs of Kramers conjugate functions $\{ \pm \Psi, \pm T \Psi\},\{ \pm i T \Psi, \pm i \Psi\}$, $\{ \pm T \Psi, \mp \Psi\}$ and $\{ \pm i \Psi, \mp i T \Psi\}$ are also KCB giving a diagonal form for $G \Sigma, \Psi$ and, if $\operatorname{rank}\left(G_{\Sigma, \Psi}\right)=r>1$, these pairs are the only ones that fulfil this condition. It is easy to check 
that these different choices of the KCB induce a sign change of two of the values of $\left(G_{X}, G_{Y}\right.$, $G_{Z}$ ) keeping the product $G_{X} G_{Y} G_{Z}=\Delta$ fixed.

As $N_{Z}$ is a traceless hermitian operator, there is a pair of Kramers conjugate states, $\{\varphi . T \varphi\}$, of eigenstates of $N_{Z}$ and the eigenspaces of $N_{Z}$ are non-degenerate $\left(G_{Z} \neq 0\right.$, see above). Then, $\Psi=e^{i \delta} \varphi$ and $T \Psi=e^{-i \delta} T \varphi$ (or $\Psi=e^{-i \delta} T \varphi$ and $T \Psi=-e^{i \delta} \varphi$ ) where $\delta$ is a real number. Now the question is to determine the $\delta$ value that produces the pair $\{\Psi, T \Psi\}$, which provides the diagonal form of $G_{\Sigma, \Psi}$.

Writting $<\varphi\left|N_{Y}\right| T \varphi>=-i|<\varphi| N_{Y}|T \varphi>| e^{i \delta_{0}}$ it follows that

$$
\left\langle\Psi\left|N_{Y}\right| T \Psi\right\rangle=-i e^{ \pm i\left(\delta_{0}-2 \delta\right)}|\varphi| N_{Y}|T \varphi\rangle=-\frac{i}{2} G_{Y}
$$

where the positive sign in the exponent corresponds to $\Psi=e^{i \delta} \varphi$ and $T \Psi=e^{-i \delta} T \varphi$ and the negative appears when $\Psi=e^{-i \delta} T \varphi$ and $T \Psi=-e^{i \delta} \varphi$.

If $\operatorname{rank}\left(G_{\Sigma, \Psi}\right)=r=1$ then $G_{Y}=0$ and consequently $<\varphi\left|N_{Y}\right| T \varphi>=0$ for any pair of Kramers conjugate eigenstates of $N_{Z}$. So, any of these pairs provide the desired KCB. On the other hand if $\operatorname{rank}\left(G_{\Sigma, \Psi}\right)=r>1$ then $G_{Y} \neq 0$ and it is a real number. Consequently, from (18) it follows that $\delta=\delta_{0} / 2+k \pi / 2$ with $k$ an integer number. The eight pairs, $\left\{e^{i \delta} \varphi, e^{-i \delta} T \varphi\right\}$ and $\left\{e^{-i \delta} T \varphi,-e^{i \delta} \varphi\right\}$ with $k=0, \ldots, 3$, of Kramers conjugates states correspond to the eight KCB given above.

In conclusion, there is, at least a reference frame, $\Sigma_{0} \equiv\left(X_{0}, Y_{0}, Z_{0}\right)$ and a $\mathrm{KCB},\left\{\Psi_{0}\right.$, $\left.\mathrm{T} \Psi_{0}\right\}$, with

$$
G_{\Sigma_{0}, \Psi_{0}}=s\left(\begin{array}{lll}
\left|G_{X}\right| & & \\
& \left|G_{Y}\right| & \\
& & \left|G_{Z}\right|
\end{array}\right)
$$

so, $G \Sigma_{\Sigma_{0}}, \Psi_{0}$ is diagonal and all its non-null element have the same sign, namely, the sign of $s$. We will talk about "a canonical description" of the KD when such a choice of the reference frame and of the KCB is used.

Let us summarize the above obtained results. Given a microscopic model of the paramagnetic entity, for any calculation method and any initial reference frame used, and once a wave-function in the ground $\mathrm{KD}, \Phi$, is obtained, the above described procedure can be applied. The reference frame $\Sigma_{0} \equiv\left(X_{0}, Y_{0}, Z_{0}\right)$, determined by the principal directions of 
$\Gamma_{\varsigma}=G_{\zeta, \Phi} \cdot G_{\zeta, \Phi}^{t}$. The KCB $\left\{\Psi_{0}, T \Psi_{0}\right\}$ is obtained as a function of the initial one $\{\Phi, T \Phi\}$. This allows determined the matrix (19) and in such a way the canonical description of the KD is provided. We will see below that this result provides a univocal way to compare the information coming from paramagnetic resonance experiments with the predictions derived from a microscopic model of the paramagnetic Kramers entity when the ground state is a well energy isolated KD.

Before doing this task it would be convenient to introduce some transformations to take advantage of the geometrical properties of the operator space as a Hilbert space (Liouville space). This is derived after the introduction of a scalar product defined as $(A, B)=$ $\operatorname{Tr}\left(A^{+} B\right)$ for any pair of operators [JE82]. It should be remembered that, with this metric, the Pauli matrices are orthogonal with norm equal to $2^{1 / 2}$. On the other hand the Pauli matrices form a basis of the real linear space of the traceless hermitian $2 \times 2$ matrices. With these considerations we built a vector operator, hereafter denoted by $\vec{n}$, in such a way that the matrix expression of its components in the $\Sigma_{0}\left(X_{0}, Y_{0}, Z_{0}\right)$ reference frame, referred to the KCB $\left\{\Psi_{0}, \mathrm{~T} \Psi_{0}\right\}$, are multiples of the Pauli matrices (by a factor $\left.1 / 2^{1 / 2}\right)$. Then, $\operatorname{Tr}\left(n^{+} n_{q}\right)=\delta_{p q}(p, q$ $\left.=X_{0}, Y_{0}, Z_{0}\right)$ and, consequently, the same holds for the components of the $\vec{n}$ vector operator in an arbitrary reference frame, $\varsigma \equiv(x, y, z)$. So, the components $\left\{n_{j}\right\}_{j=x, y, z}$ are an orthonormal basis of the real space of the traceless Hermitian operators acting on the $\mathrm{KD}$, that coincide with the time-odd Hermitian operators (see section II). Note that the vector operator $\vec{n}$ acts within the physical states of the KD and:

$$
N_{r}=\frac{S}{\sqrt{2}}\left|G_{r}\right| n_{r} \quad\left(r=X_{0}, Y_{0}, Z_{0}\right)
$$

Then, the components of the $\vec{N}$ vector operator, defined in equation (1), are expressed as a function of the $\vec{n}$ components in an arbitrary reference frame. Let $P \in \mathbf{S O}(3)$ be the direct orthogonal matrix that relates a $\varsigma \equiv(x, y, z)$ reference frame with the $\Sigma_{0}\left(X_{0}, Y_{0}, Z_{0}\right)$ one, see equation (14). Defining:

$$
\gamma_{\Sigma_{0}}=\left(\begin{array}{lll}
\left|G_{X}\right| & & \\
& \left|G_{Y}\right| & \\
& & \left|G_{Z}\right|
\end{array}\right)
$$

and taking into account the vector properties of $\vec{N}$ and $\vec{n}$, it follows that: 


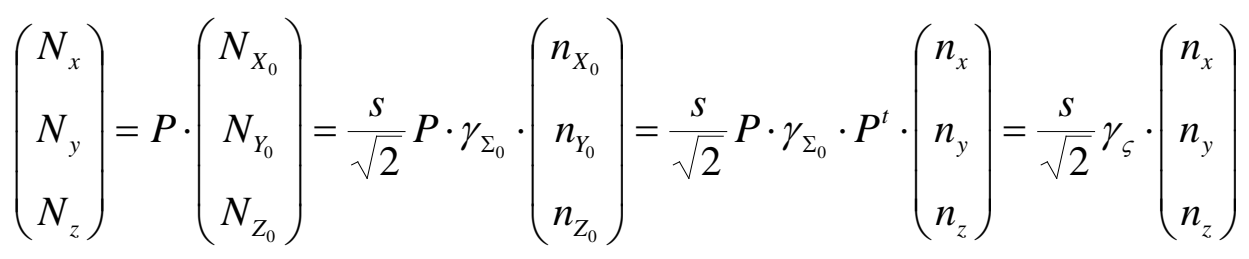

with

$$
\gamma_{\varsigma}=P \cdot \gamma_{\Sigma_{0}} \cdot P^{t}
$$

This let us introduce the $\tilde{\gamma}$ tensor, whose matrix expression in any reference frame, $\varsigma$ $\equiv(x, y, z)$, is given by the previous equation. Note that, despite of the similarities of equations (19) and (21) there is a deep difference between $G_{\Sigma_{0}, \Psi_{0}}$ (the of the $G$-matrix expression for the particular reference frame and KCB providing a canonical description of the KD) and $\gamma_{\Sigma_{0}}$ (the matrix expression of the tensor $\tilde{\gamma}$ in the reference frame $\Sigma_{0}$ ). Then, the following tensor relation between the $\vec{N}$ and $\vec{n}$ vector operators holds:

$$
\vec{N}=\frac{s}{\sqrt{2}} \tilde{\gamma} \cdot \vec{n}=\frac{1}{\sqrt{2}} \tilde{g} \cdot \vec{n}
$$

where

$$
\tilde{g}=s \tilde{\gamma}
$$

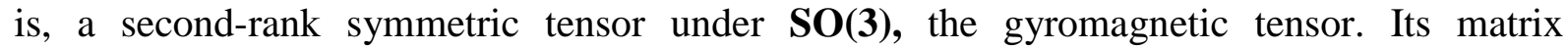
expression in the $\Sigma_{0} \equiv\left(X_{0}, Y_{0}, Z_{0}\right)$ reference frame is diagonal and it coincides with the canonical form of $G_{d}$. Therefore, all its diagonal elements have the same sign and it coincides with $s$, the sign of $\operatorname{det}(G)$.

In this way (1) is given by

$$
H_{\text {Ze }}=\mu_{B} \vec{B} \cdot\left(\vec{L}+g_{e} \vec{S}\right)=\mu_{B} \vec{B} \cdot \tilde{g} \cdot\left(\frac{1}{\sqrt{2}} \vec{n}\right)
$$

which act on the physical wave functions in the KD.

As it is formally discussed in Supplementary Information (section S.1) the behaviour of the KD under an applied magnetic field, $\vec{B}$, which is described by (26) is reproduced by the behaviour of an $S=1 / 2$ system described by the Hamiltonian:

$$
H_{Z e}^{S}=\mu_{B} \vec{B} \cdot \tilde{g} \cdot \vec{S}
$$

Moreover, any physical prediction on the physical KD coincides with that made on this $S=1 / 2$ system if the substitution rule

$$
\vec{n} \leftarrow \sqrt{2} \vec{S}
$$


see (S.2), is applied. Then, equation (27) gives a fictitious spin-Hamiltonian suitable for describing the magnetic behaviour of the KD.

It is known that in the case of an $S=1 / 2$ system the EPR spectrum and its angular variation (when a single crystal is available) supply information about the $\tilde{g}$-tensor [PF72]. As the most of spectrometers has not available a circular polarised microwave source only information about the absolute value of the $g$-tensor principal values and the orientation of its principal axes (which define the $\Sigma_{0}$ reference frame) respect to the laboratory axes is obtained. If the crystal is properly oriented this information is directly translated to the orientation of the $\Sigma_{0}\left(X_{0}, Y_{0}, Z_{0}\right)$ reference frame with respect to the initial reference systems implicitly used for calculating the magnetic properties of the $\mathrm{KD}$ in the suitable microscopic model (see above). This type of experiment performed without using circular polarised microwave will be designed hereafter as “conventional EPR experiment”.

On the other hand, the sign of the determinant of the $g$-tensor, which coincide with the product of the three principal $g$-values, can be determined with a spectrometer having a circular polarised source is used, by measuring the difference between the spectra taken with opposite polarizations [AB70b, PO83]. Therefore, if a single crystal is available and the angular variation of the spectrum is measured using circular polarised microwaves (we say that we do a "complete EPR experiment”) the canonical form of the $G$-matrix, see eq. (19) and the $\Sigma_{0}\left(X_{0}, Y_{0}, Z_{0}\right)$ reference frame can be experimentally determined and, using the protocol indicated above, it can be straightforward compared with the predicted values.

So, a univocal bridge among the experimental findings and the theoretical predictions has been given, which overcomes the problems derived from the sign ambiguity. When only data from a conventional EPR (without circular polarised microwave soured) experiment are available, there will be an uncertainty in the sign of $\operatorname{det}(G)$.

\section{Point SYMMETRY AND THE $g$-TENSOR}

The role of the symmetry in the EPR spectroscopy has been recognized from its early times and the analysis of the rotational diagram is a powerful tool for getting information about the local symmetry of the paramagnetic entities [PF72b, PE73, SN92, WE07, BG09]. In this context it is worth noting that the approaches adopted by several authors to overcome the sign ambiguity were based on symmetry considerations [GA98b, CC08]. Here we will briefly analyse how the symmetry properties of the paramagnetic entity translate straightforwardly 
into the $g$-tensor (and into the $G$-matrix) as defined in the previous section and, consequently, this information can be obtained experimentally.

Firstly, it is important to remember that both, the magnetic field and the $\vec{N}$ operator, behave as vectors under the $\mathbf{S O}(3)$ but remain invariant under inversion. In this way, the transformation properties of the Zeeman interaction (1) under SO(3) operations have been used to define the $g$-tensor. However, the point group, $\mathbf{G}$, describing the local symmetry of the paramagnetic entity is not, in general, a subgroup of $\mathbf{S O ( 3 )}$ since $\mathbf{G}$ could include improper rotations. This drawback can be overcome by using, instead of $\mathbf{G}$, the point group $\hat{\mathbf{G}}$, a subgroup of $\mathbf{S O}(\mathbf{3})$ that coincides with $\mathbf{G}$ if all its elements are proper rotations. $\hat{\mathbf{G}}$ is either a subgroup of $\mathbf{G}$ (of index two) if the inversion is a symmetry element of $\mathbf{G}$, or it is isomorphic to $\mathbf{G}$ (see appendix B for details about how $\hat{\mathbf{G}}$ is built). In the following we shall restrict ourselves to transformation operations of $\hat{\mathbf{G}}$.

Let $R$ be a direct orthogonal matrix that represents a transformation of $\hat{\mathbf{G}}$. Obviously, $R^{t}$ it also a direct orthogonal matrix belonging to $\hat{\mathbf{G}}$. $R^{t}$ can be also seen as a rotation matrix of the reference frame. On the other hand, from (3)

$$
H_{Z e}=\mu_{B} B\left(u^{t} \cdot N\right)=\mu_{B} B\left\{(R \cdot u)^{t} \cdot N\right\}=\mu_{B} B\left\{u^{t} \cdot\left(R^{t} \cdot N\right)\right\}=\mu_{B} B\left(u^{t} \cdot \breve{N}\right)
$$

where $\breve{N}=R^{t} \cdot N$ or equivalently $N=R \cdot \breve{N}, N$ being a column matrix, whose elements are the components of the vector operator $\vec{N}$ in the given reference frame. $\breve{N}$ is defined in a similar way and, consequently, $G_{\zeta, \Phi}=R \cdot \breve{G}_{\zeta, \Phi}$ for any reference frame and any KCB.

As we have seen in section 3 , if $P$ is the orthogonal matrix that makes $\Gamma_{\varsigma}=G_{\zeta, \Phi} \cdot G_{\zeta, \Phi}^{t}$ diagonal, see (17), it follows that $P \cdot R$ makes $\breve{\Gamma}_{\varsigma}=\breve{G}_{\varsigma, \Phi} \cdot \breve{G}_{\varsigma, \Phi}^{t}$ diagonal with the same eigenvalues since $\Gamma_{\varsigma}=R \cdot \breve{\Gamma}_{\varsigma} \cdot R^{t}$. Moreover, the columns of $P$ and, hence, the columns of $P \cdot R$ correspond to the principal directions of $\Gamma_{\varsigma}$. Note that the columns of $P \cdot R$ give the coordinates of the columns of $P$ by the transformation $R$, and they correspond to the same eigenvalues. Besides, $R$ is a direct rotation. Then, if $R$ is an $n$-fold rotation, with $n>2$, there are only two possibilities: the rotation axis coincides with one of the principal directions of $\Gamma_{\sigma}$ or two eigenvalues are equal and their principal directions are perpendicular to the rotation axis.

In consequence, since in the canonical description of the Kramers doublet the diagonal expression of $G_{\Sigma_{0}, \Psi_{0}}$ is given by positive square roots of the eigenvalues of $\Gamma_{\sigma}$ together with the sign of $\operatorname{det}(G), s$, it follows that the existence of a $n$-fold rotation axis, with $n>2$, implies 
the equivalence of two of these diagonal elements of $G_{\Sigma_{0}, \Psi_{0}}$. Also, the reference frame $\Sigma_{0} \equiv$ $\left(X_{0}, Y_{0}, Z_{0}\right)$ is determined in such a way that one axis, commonly the $Z_{0}$ axis, is along the $n$ fold rotation axis and the other two are any pair of arbitrary directions normal to this direction. If we are dealing with a cubic symmetry, octahedral, cubal or tetrahedral environment, it follows straightforwardly that all diagonal element of $G_{\Sigma_{0}, \Psi_{0}}$ are equal. The construction of the $g$-tensor, as presented in the previous section, guarantees that it fulfils the same symmetry properties.

\section{LIGAND FIELD AND THE $g$-TENSOR}

As follows from section 3, if the electronic structure of the KD is known (whatever the degree of sophistication adopted in the calculation) a $g$-tensor can be defined in a univocal way, which can be compared with the results derived from a complete set of EPR experiments. Conversely, attempts to obtain information about the electronic structure of the paramagnetic impurity from the experimentally determined $g$-tensor have repeatedly appeared in the literature. In contrast, Rudowicz and Sung [RS01] wrote "The electron magnetic resonance techniques can measure only the ZFS/FS (fine structure) parameters, whereas the CF/LF (ligand field) parameters can be determined by optical spectroscopy, neutron scattering, and/or Raman spectroscopy”.. This issue has been discussed by Rudowicz and coworkers in depth [RS01, RU08a, RU08b, RK14, RK15, RK15b]. The misuse of the CF/LF and ZFS/FS terms often appearing in the literature is attributed as the main source of errors.

In the present case, as we are dealing with a Kramers doublet, no ZFS/FS contribution is present. Hence, the following discussion will be focused in the attempt of getting information of the ligand field from the measured $g$-tensor. This will be possible only in some specific cases.

However, if the ligand field contribution is the main one giving out a non-degenerate ground term and the spin-orbit contribution can be considered up to a first order, the approach by Pryce [PR50] and Abragam and Pryce [AP51] indicates that the deviations of $g$-tensor principal values from the free electron one are of the order of $\xi / \Delta E$, $\xi$ being the spin-orbit coupling constant and $\Delta E$ the energy of any excited terms relative to the ground term. Hence, the experimentally determined principal $g$-values can provide an estimate for the ligand field splitting given by $\Delta E$ when the quotients $\xi / \Delta E$ are small enough. The question now is to analyse what happens when this perturbation scheme is no longer valid because the spin-orbit 
contribution is similar to the ligand field one. In this situation there could be a substantial entanglement of the orbital and spin wave functions in the states of the ground $\mathrm{KD}$, whose magnetic properties are described with a fictitious spin-Hamiltonian with a $g$-tensor that could depart significantly from the free electron $g$-value. Therefore, it is unclear if a dependence of the $g$-tensor with the ligand field contribution parameters can be univocally established.

Let us analyse this point by considering a simple example, which, in spite that it could be rather academic, will be useful for clarifying those ideas. Here we only introduce the model and discuss the relevant conclusions. The cumbersome technical details are given as Supplementary Information (see S.2)

Let us consider a single electron occupying a $p$-orbital in an environment given by two unlike pairs of ligands placed in the corners of a square in trans positions (see figure 1.a). The reference frame, $\Sigma \equiv(X, Y, Z)$, we will use is also given in Figure 1.a. Due to the ligand field effect the $p$ orbital degeneracy is lifted. Let the $p_{z}$ orbital be the ground orbital. As a convention this $p_{z}$ orbital will be taken as the origin of energy, $(E=0)$. So, the relevant energies can be given as a function of the $\Delta_{X}$ and $\Delta_{Y}$ parameters (see figure 1.b). With this convention $\Delta_{X} \geq \Delta_{Y} \geq 0$.

When the electronic spin is considered the ground state is two-fold degenerate and it results in a KD. As shown in Supplementary Information, S.2, a KCB is given by:

$$
\begin{gathered}
\Psi^{+}=u\left|p_{Z}, \alpha+i v\right| p_{Y}, \beta+w \mid p_{X}, \beta \\
\left.\Psi^{-}=u\left|p_{Z}, \beta+i v\right| p_{Y}, \alpha\right\rangle+w \mid-p_{X}, \alpha
\end{gathered}
$$

where the $(u, v, w)$ coefficients are chosen to be real with $u^{2}+v^{2}+w^{2}=1$. They accomplish:

$$
\frac{E}{\xi}=-\frac{1}{2} \frac{v+w}{u} \quad \frac{\Delta_{Y}}{\xi}=\frac{1}{2}(u+v+w)\left(\frac{1}{v}-\frac{1}{u}\right) \quad \frac{\Delta_{X}}{\xi}=\frac{1}{2}(u+v+w)\left(\frac{1}{w}-\frac{1}{u}\right)
$$

Moreover, since $\Delta_{X} / \xi \geq \Delta_{Y} / \xi \geq 0$,

$$
u>v \geq w \geq 0
$$

Using the KCB given in (30) and the reference frame $\Sigma \equiv(X, Y, Z)$ depicted in figure 1.a the $G_{\Sigma, \Psi}$ matrix is calculated. It results to be diagonal, with: 


$$
\begin{aligned}
& G_{X X}=2\left(u^{2}+v^{2}-w^{2}-2 u v\right)=2\left\{(u-v)^{2}-w^{2}\right\}=2(u-v-w)(u-v+w) \\
& G_{Y Y}=2\left(u^{2}-v^{2}+w^{2}-2 u w\right)=2\left\{(u-w)^{2}-v^{2}\right\}=2(u-v-w)(u+v-w) \\
& G_{Z Z}=2\left(u^{2}-v^{2}-w^{2}+2 v w\right)=2\left\{u^{2}-(v-w)^{2}\right\}=2(u-v+w)(u+v-w)
\end{aligned}
$$

where we have taken $g_{e}=2$. Besides,

$$
\operatorname{det}(G)=G_{X X} G_{Y Y} G_{Z Z}=8(u-w-w)^{2}(u-v+w)^{2}(u+v-w)^{2} \geq 0
$$

So, in any case, the principal $g$-values are given by:

$$
g_{X}=\left|G_{X X}\right| \quad g_{Y}=\left|G_{Y Y}\right| \quad g_{Z}=G_{Z Z}
$$

and the principal axes of the $g$-tensor coincide with the formerly defined $\Sigma \equiv(X, Y, Z)$ reference frame.

Consequently, the former description is canonical if $G_{X X}, G_{Y Y}, G_{Z Z}$ are all positive (or zero). Taking into account (32), it follows that $G_{Z Z}$ is always positive and $G_{X X}$ and $G_{Y Y}$ have the same sign, which coincide with the sign of $(u-v-w)$. As $u, v, w$ are continuous functions of $\Delta_{X} / \xi$ and $\Delta_{Y} / \xi$, see Supplementary Information, S.2, the condition $(u-v-w)=0$ will define the regions of the $\left(\Delta_{X} / \xi, \Delta_{Y} / \xi\right)$ plane where the previous description is canonical or not. From (31), the condition $(u-v-w)=0$ is equivalent to $\Delta_{X} \Delta_{Y}=\xi$. So, the previous description is canonical if $\Delta_{X} \Delta_{Y}>\xi$ whereas non-canonical if $\Delta_{X} \Delta_{Y}<\xi$. This is illustrated in figure 2.

Let us assume for the moment that the above description is canonical, $(u-v-w)>0$ and $G_{X X}, G_{Y Y}, G_{Z Z}$ are all positives. Then $G_{X X}=g_{X}, G_{Y Y}=g_{Y}$ and $G_{Z Z}=g_{Z}$ with $g_{X} \leq g_{Y} \leq g_{z}$. Once these values are determined experimentally, the $(u, v, w)$ parameters can be calculated from (33) and then the LF parameters relative to the spin-orbit constant, $\Delta_{\chi} / \xi$ and $\Delta_{y} / \xi$, are obtained from (31). In the opposite case, $G_{X X}=-g_{X}, G_{Y Y}=-g_{Y}$ and $G_{Z Z}=g_{Z}$ also with $g_{X}$ $\leq g_{Y} \leq g_{Z}$, so we can proceed in the same way. However, EPR experiments cannot provide any information about the condition LF parameters actually fulfil. So, there is no way to distinguish among the two formerly described situations through EPR spectroscopy since in both cases $g_{x} g_{x} g_{z} \geq 0$. This outcome illustrates the observation by Rudowicz and Sung [RS01] that EPR spectroscopy is not, in general, a suitable tool for gathering information about CF/LF parameters.

\section{SOME EXAMPLES FOR THE LITERATURE}


Next, we go to present the application of the former description to some cases that can be found in the literature. We include low spin heminic system, some $d^{7}$ ions in a strong ligand field situation and Kramers trivalent rare-earth. The examples are necessarily no comprehensive but we expect it will help to show the general validity of the formalism given in section 3.

\section{1. $d^{5}$ ions in LS configuration (strong ligand field)}

Let start with the description of the EPR properties of the $d^{5}$ ions in the low spin configuration. These systems, especially LS - Fe $\mathrm{F}^{\mathrm{III}}$ ions, have received particular attention because many hemoproteins show such behaviour [BS03, WW99, WA04, WA99, AM07, ZB09]. It is worth mentioning that in those works it was implicitly admitted that each principal value of the $g$-tensor has a definite sign in spite that it cannot be determined from the EPR spectra since these experiments only provides the magnitude of those $g$-factors. Then, different strategies were proposed to circumvent this problem that, herein, will be called the "sign problem".

A first description of the electronic structure of the ground state Kramers doublet of this system was given by Bleaney and O’Brian [BB56], who established a relation between the calculated and the observed gyromagnetic factors. They also introduced the "sign problem". A year later Griffith used the same approach to derive the electronic structure of $\mathrm{Fe}^{\mathrm{III}}$ in ferrihaemoglobin azide [GR57] from its EPR spectrum previously reported [GI57]. This author obviated the sign problem when relating the calculated with the experimental absolute values and an implicit (positive) sign was assigned to all principal $g$-values in the same paper when the ligand field splitting was estimated. It is worth noting that the states describing the ground doublet show noticeable entanglement between the orbital and spin wave functions and, consequently, the electronic spin is not a good quantum number. Hence, the spin introduced in this SH description is actually a fictitious spin [RU87].

Later, Taylor [TA77] derived his well-known equations, which relate the principal $g$ values with the coefficients used for describing the ground state wave function. Those coefficients are related to the splitting of the $t_{2 g}$ orbitals due to the low symmetry ligand field and the "sign problem" is also discussed. Since then, Taylor's equations have been widely used; see references [BO77, RI94, GA98a, WA99, BS03, ZB09] where some reviews are presented. Among them a contribution by McGarvey [GA98b] developed an extension of the 
former approaches by including the configuration interaction up to second order in the spinorbit interaction. This is necessary for $4 d^{5}, \mathrm{Ru}^{\mathrm{III}}$, and $5 d^{5}$, Os ${ }^{\mathrm{III}}$, systems because of their higher spin-orbit coupling constants. The "sign problem” was also tackled by using symmetry arguments, it is also noted that these arguments could be inexact if the symmetry departs significantly from the octahedral one. However, the "sign problem" persists in the literature [RI94, WA99, WA04, ZB09]. In a recent paper [AM07] the Taylor's formulation was revised and it was noted that additional information about the sign of the gyromagnetic factors can be obtained from knowledge of the ${ }^{57}$ Fe hyperfine coupling tensor derived from Mössbauer data.

As the ground state of these LS- $d^{5}$ ions is a KD the formalism given in section 3 can be applied to determine their magnetic behaviour. This task is given in Supplementary Information (section S.3) by using the LF description given in [AM07]. The results are similar to those found in the case of the example given in section 5 but in this case all the ambiguities are overcome when the sign of the product of the three principal $g$-values (which coincides with the sign of the determinant of the matrix $G$ ) is known. So, the "sign problem" in these LS- $d^{5}$ systems need to be rethought. In terms of section 3 formulation, the individual signs actually correspond to the sign of diagonal components of a diagonal $G$-matrix (eq. 13) but those signs have not a physical meaning as far as they depend on the chosen KCB. So, the "sign problem" is not a real problem but a fanciful one.

If the sign of the product of the three principal $g$-values is not experimentally determined as it usually happens (when a conventional EPR experiment using linear polarized microwave is performed) two possibilities arise for the LF splitting ( $\Delta$ and $V$ parameters, see figure S.1). In general, see figure S.2, those pairs of values are very different. So, some additional physical arguments could help to distinguish among them.

\subsection{LS- $d^{7}$ ions in tetragonal symmetry with donor ligands}

An early interpretation of the spin Hamiltonian parameters used for the description of the EPR spectra of $\mathrm{Co}^{\mathrm{II}}\left(d^{7}\right)$ in an environment close to the square-planar one was given by Girffith [GR58]. A dominant ligand field was assumed and the spin-orbit interaction was introduced up to first order, which causes a mixing of the excited states in the ground state wave function. The same approximation was followed by other authors [BM70, BC73]. A work by McGarvey [GA75] deserves special mention as it presents a comprehensive model to analyse the magnetic properties of these "low spin" $d^{7}$ systems. Since then, several 
descriptions of the electronic properties of the ground state doublet of this LS- $d^{7}$ configuration have appeared [MR80, BB82, SM07]. In particular, Nishida and Kida [NK78] proved that a good estimation of the $g$-tensor is achieved by neglecting the spin doublets that involve an unpaired electron in the highest energy $x^{2}-y^{2}$ orbital. So, seven electron have to be accommodated in the four low-laying orbitals and the hole formalism can be used for describing the electronic properties of the ground state.

Recently, we have used a similar approximation to study the magnetic properties of homoleptic organoderivates of $\mathrm{Ni}(\mathrm{II})$ [AA09] and $\mathrm{Co}(\mathrm{II})$ [GA14] paramagnetic complexes of general formula $\left[\mathrm{M}\left(\mathrm{C}_{6} \mathrm{X}_{5}\right)_{4}\right]^{-\mathrm{q}}, \mathrm{M}=\mathrm{Ni}(\mathrm{III}), \mathrm{Co}(\mathrm{II})$, where $\left(\mathrm{C}_{6} \mathrm{X}_{5}\right)$ stands for a perhalophenyl radical ( $\mathrm{X}=\mathrm{F}$ or $\mathrm{Cl}$ ). In these cases the metal environment has a symmetry close to $\mathbf{D}_{4}$, see reference [AA09, GA14], and the observed EPR spectra were axial. As discussed in the above cited references there are two possibilities for the ordering of the single-electron energy levels in a SP-4 (planar-square) environment. In both of them the $b_{1}\left(\mathrm{x}^{2}-\mathrm{y}^{2}\right)$ orbital is strongly destabilised while the $e(x z, y z)$ doublet have the lowest energy. The $(x, y, z)$ reference frame is defined with the $z$ axis along the four-fold rotation axis and the equivalent $x$ and $y$ axes are given by the metal-ligand bond directions. The actual ordering of the $a_{1}\left(z^{2}\right)$ and $b_{2}(x y)$ determines the SOMO orbital and, consequently, the magnetic properties of the complex. (A) and (B) labels were introduced for the two cases of the unpaired electron in a $a_{1}\left(z^{2}\right)$ and $b_{2}(x y)$ orbital, respectively. The LF energy of the SOMO orbital measured from the lowest energy $e(x z, y z)$ doublet, will be denoted by $\Delta$. This is summarized in Figure 3.

For the case (A), a KCB for the ground (hole) KD is given by:

$$
\begin{gathered}
\Phi_{A}=\cos \theta\left|\psi_{z} \alpha\right\rangle-\frac{1}{\sqrt{2}} \sin \theta\left|d_{x z} \beta\right\rangle-\frac{i}{\sqrt{2}} \sin \theta \mid d_{y z} \beta \\
T \Phi_{A}=\cos \theta\left|\psi_{z} \beta\right\rangle+\frac{1}{\sqrt{2}} \sin \theta\left|d_{x z} \alpha\right\rangle-\frac{i}{\sqrt{2}} \sin \theta \mid d_{y z} \alpha
\end{gathered}
$$

where some $s$ contribution in the SOMO orbital $\psi_{z}$ has to be considered; $\psi_{z}=a d_{z 2}-b s$, where $a, b$ are real coefficient satisfying $a^{2}+b^{2}=1 \cdot \operatorname{tg}(2 \theta)=2 \sqrt{6} a \zeta /(2 \Delta+\zeta)$ being $\zeta$ the singleelectron spin-orbit coupling constant and $\Delta$ the energy of the SOMO measured from the lowest energy $e(x z, y z)$ doblet, see figure 3 , with $0 \leq \theta \leq \pi / 4$.

Similarly, for the case (B) a KCB of the hole ground state is: 


$$
\begin{gathered}
\Phi_{B}=\cos \theta\left|d_{x y} \alpha\right\rangle+\frac{i}{\sqrt{2}} \sin \theta\left|d_{x z} \beta\right\rangle+\frac{1}{\sqrt{2}} \sin \theta \mid d_{y z} \beta \\
T \Phi_{B}=\cos \theta\left|d_{x y} \beta\right\rangle+\frac{i}{\sqrt{2}} \sin \theta\left|d_{x z} \alpha\right\rangle-\frac{1}{\sqrt{2}} \sin \theta \mid d_{y z} \alpha
\end{gathered}
$$

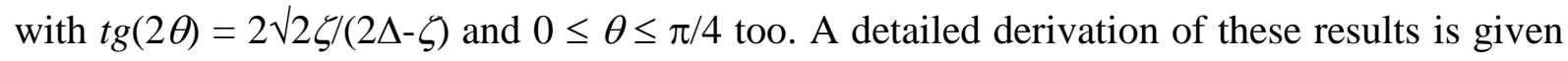
in [AA09, GA14].

For applying the formalism given in section 3 we calculate the $G$-matrix, see eq. (5), using the KCB and the reference frame $(x, y, z)$ above introduced. If an orbital reduction is modelled by a parameter $k$ [AA09, GA14], we obtain:

$$
G^{A}=\left(\begin{array}{lll}
1+\cos 2 \theta+\sqrt{6} k a \sin 2 \theta & & \\
& 1+\cos 2 \theta+\sqrt{6} k a \sin 2 \theta & \\
& (2-k) \cos 2 \theta+k
\end{array}\right)
$$

in the case (A), and

$$
G^{B}=\left(\begin{array}{lll}
1+\cos 2 \theta+\sqrt{2} k \sin 2 \theta & & \\
& 1+\cos 2 \theta+\sqrt{2} k \sin 2 \theta & \\
& & (2+k) \cos 2 \theta-k
\end{array}\right)
$$

in the case (B). It has been taken $g_{e}=2$ in the two former expressions.

In both case, the $G$-matrix results to be diagonal with $G_{X X}=G_{Y Y}$, which is a consequence of the $S P-4$ symmetry. Then the sign of $\operatorname{det}(G)$ is given by the sign of $G_{z z}$. Taking into account that $0 \leq \theta \leq \pi / 4$ in both cases, it results that $G_{z z}>0$ for usual values of $k$ (close to one).

Previous studies took no care on the sign of principal $g$-values [GR58, GA75, BM70, BC73, MR80, NK78, BB82, SM07, AA09, GA14] and gave the comparison between calculations and observations in terms of absolute (positive) value. As we show here , incidentally, this unjustified choice coincides with the actual canonical description.

\subsection{Rare earths ions with an odd number of unpaired electrons}

The first description of the magnetic properties of Kramers rare-earth ions was given by Elliot and Stevens [ES52, ES53]. Those results were restricted to axial (trigonal) symmetry 
and later were extended to a more general symmetry by Rubins [RU70a, RU70b]. A detailed presentation was given in the Abragam and Bleaney book [AB70d] and, more recently, in [MI11] where the method developed in [MC97] is summarized. On the other hand there is a great amount of published papers on the EPR characterization of Kramers rare-earth ions in functional materials mainly with the aim to discriminate between the different occupancy sites, which drive its behaviour [KE02]. Some example was given in [AS71] and more recently in [YH01, GK02, GG06, ML09]. The potential use of these systems in quantum information processing [LS12, AL15] gives a renewal interest to their study.

Due to the inner character of the $f$ electrons the effect of LF can be consider as a first order perturbation within the free ion levels characterized by the total angular momentum quantum number $J$. In low enough symmetry the energy levels are KD and their energy separation is about tenths or hundreds of reciprocal wavenumbers. At low enough temperature only the ground $\mathrm{KD}$ is populated and, consequently, the magnetic properties can be described by using an effective $S^{\prime}=1 / 2$ spin. Hence, the electronic Zeeman interaction is characterized by a $g$-tensor (or a $g$-matrix) and, in most of the published papers, the comparison between the experimental determination and the calculations of the principal $g$-factors is given in terms of absolute values. This was explicitly indicated in the papers by Elliot and Stevens [ES52, ES53]. Later Robins [RU70a, RU70b] resigned to calculate the sign of the principal $g$-factors. This last author indicated that all of them can be arbitrarily taken positive forgetting that the sign of the product can be experimentally determined [PR59]. This position remains nowadays [MI11] with few exceptions found in the literature. In a previous study of the EPR spectrum of $\mathrm{Er}^{3+}$ in $\mathrm{Y}_{2} \mathrm{SiO}_{3}$ single-crystals [GG06] it is claimed that sign of the $g$-matrix can be univocally determined by fitting the angular variation of the spectrum due to even isotopes $(I=0)$ along three orthogonal planes, forgetting that this experiment only provides information about the " $g$ tensor" [PF72].

Since the ground state of those systems is a KD the formalism given in section 3 can be directly used as it is briefly sketched below.

After modelling LF with a suitable potential, which implicitly assumes the choice of a particular reference frame, $\sigma \equiv(x, y, z)$, a KCB of the ground $\mathrm{KD}$ can be expressed as:

$$
\Phi=\sum_{M=-J}^{J} c_{M}|J, M\rangle \quad T \Phi=\sum_{M=-J}^{J}(-1)^{J+M} C_{-M}^{*} \mid J, M
$$


where the $c_{M}$ coefficients are determined by the diagonalization of the LF contribution within the ground energy level of the free ion [RU70a, RU70b]. On the other hand, if a magnetic field, $\vec{B}$, is applied, the Zeeman interaction restricted to this energy level of the free ion is given by

$$
H_{Z e}=\mu_{B} g_{J} \vec{B} \cdot \vec{J}
$$

where $g_{J}$ is the Landé factor of the free atom level. So, see eq (1), $\vec{N}=g_{J} \vec{J}$ and, using the KCB (40), the $G$-matrix (see eq. 5) results to be

$$
G_{\Phi, \sigma}=g_{j} \sum_{M=-J}^{J} G_{\zeta, \Phi}^{M}
$$

where

$$
G_{\zeta, \Phi}^{M}=\left(\begin{array}{ccc}
(-1)^{J+M}\{M\} \operatorname{Re}\left(C_{M+1} C_{-M}-C_{M} C_{-(M+1)}\right) & -(-1)^{J+M}\{M\} \frac{1}{2} \operatorname{Im}\left(C_{M+1} C_{-M}-C_{M} C_{-(M+1)}\right) & 2\{M\} \operatorname{Re}\left(C_{M} C_{M+1}^{*}\right) \\
-(-1)^{j+M}\{M\} \operatorname{Im}\left(C_{M+1} C_{-M}+C_{M} C_{-(M+1)}\right) & (-1)^{J+M}\{M\} \operatorname{Re}\left(C_{M+1} C_{-M}+C_{M} C_{-(M+1)}\right) & 2\{M\} \operatorname{Im}\left(C_{M} C_{M+1}^{*}\right) \\
(-1)^{J+M} 2 \operatorname{Re}\left(C_{M} C_{-M}\right) & (-1)^{M} 2 \operatorname{Im}\left(C_{M} C_{-M}\right) & 2 M\left|C_{M}\right|^{2}
\end{array}\right)
$$

with $\{M\}=[J(J+1)-M(M+1)]^{1 / 2}$.

The sign of the principal $g$-factors is given by the sign of determinant of the $G$-matrix (E.33) while their absolute values corresponds to the positive square root of the eigenvalues of $G_{\Phi, \sigma}^{t} G_{\Phi, \sigma}$. Its eigenvectors determine the principal axes of the $g$-tensor respect to the reference frame, $\sigma \equiv(x, y, z)$, initially introduced.

Then, the ambiguities of the $g$-tensor definition in the case of rare-earth can be removed. This point is of particular relevance when studyimg pairs of weakly interacting rareearth since, as we will see in the next section, an adequate definition of individual $g$-tensor allows relating the phenomenological coupling parameters with the physical interaction in a straightforward way.

\section{SPIN HAMILTONIAN DESCRIPTION OF TWO WEAKLY COUPLED KRAMERS} DOUBLETS UNDER AN APPLIED MAGNETIC FIELD

Finally, an extension of the previously developed fictitious spin formalism will be presented, which is suitable for describing the magnetic properties associated to the lowest lying states in the case of a pair of coupled Kramers ions when the inter-ion as well as the 
individual Zeeman interactions are small enough in comparison to the energy splitting between the Kramers doublets of isolated ions. Here a general solution will be given and a fictitious spin Hamiltonian in the multispin scheme [MG10a, MG10b, MP10, MG13, MC14, RM14, RK15b] will be derived. It will include the Zeeman interaction of two isolated $S=1 / 2$ fictitious spins described by the corresponding $g$-tensors and an exchange interaction between them. This exchange interaction, written as a function of the true interaction, includes the isotropic, symmetric traceless and antisymmetric (Dzyaloshinskii-Moriya) terms. A particular solution for this problem was given a decade ago in [PT03] and this kind of Hamiltonian had been introduced in a phenomenological way on several occasions [BG90, KA93, BO99].

When two Kramers systems, (a) and (b), are coupled through an interaction, $H_{\text {int }}$, weak enough, in the absence of an applied magnetic field, the Hamiltonian of the whole system is given by:

$$
H=H_{a}+H_{b}+H_{\text {int }}=H_{0}+H_{\text {int }}
$$

where $H_{0}$ is the zero-order Hamiltonian for non-interacting systems and $H_{\text {int }}$ is considered a perturbation. If the solutions of $H_{r}\left|\phi_{r}\right\rangle=E_{r}\left|\phi_{r}\right\rangle(r=a, b)$ are known, the zero order solutions of (43) are given by $|\Psi\rangle=\left|\phi_{a}\right\rangle \otimes\left|\phi_{b}\right\rangle$ with energy $E=E_{a}+E_{b}$.

If no extra degeneracy apart from the Kramers one exists, the energy levels of each isolated subsystem will be Kramers doublets and a $\mathrm{KCB}\left\{\Phi_{r}, T_{r} \Phi_{r}\right\}$ can be chosen in each doublet of the subsystem $r(r=a, b)$ where $T_{r}$ is the corresponding time-reversal operator and $\Phi_{r}$ is any arbitrary KD state. Consequently, in such a case, the zero-order levels of the whole system are fourfold degenerate and $\left\{\left|\Phi_{a}\right\rangle \otimes\left|\Phi_{b}\right\rangle,\left|\Phi_{a}\right\rangle \otimes\left|T_{b} \Phi_{b}\right\rangle,\left|T_{a} \Phi_{a}\right\rangle \otimes\left|\Phi_{b}\right\rangle,\left|T_{a} \Phi_{a}\right\rangle \otimes\left|T_{b} \Phi_{b}\right\rangle\right\}$ is a basis of each zero-order level. In the following the shortened notation $\mid \phi_{a}$, $\left.\phi_{b}\right\rangle$ will be used for $\left|\phi_{a}\right\rangle \otimes\left|\phi_{b}\right\rangle$.

Assuming that the interaction $H_{\text {int }}$ can be considered up to a first order, it has to be applied within any of these quartets. It is important to note that $H_{\text {int }}$ is a time-even operator and the time-reversal operator of the whole system is given by $T=T_{a} \otimes T_{b}$, where $T_{a}$ and $T_{b}$ are the time-reversal operators for the subsystem (a) and (b), respectively [ME64b]. Then $T H_{\text {int }}=$ $H_{\text {int }} T$. Also, it can be assumed that $H_{\text {int }}$ is traceless by including the diagonal contributions in $H_{0}$. That allows us to express $H_{\text {int }}$, using a particular reference frame $\varsigma \equiv(x, y, z)$ and the vector operators $\vec{n}_{a}$ and $\vec{n}_{b}$ defined in section 3 for both subsystems, as 


$$
H_{i n t}=\frac{1}{2} \sum_{i, j=x, y, z} H_{i j}\left(n_{a ; i} \otimes n_{b ; j}\right)=\frac{1}{2} \sum_{i, j=x, y, z} H_{i j}\left(n_{a ; i} \otimes I_{b}\right)\left(I_{a} \otimes n_{b ; j}\right)=\frac{1}{2} \sum_{i, j=x, y, z} H_{i j} n_{i}^{(a)} n_{j}^{(b)}
$$

where the coefficient $1 / 2$ is introduced for convenience and the shorthand notation $n_{i}^{(a)}=$ $n_{a ; i} \otimes I_{b}$ and $n_{j}^{(b)}=I_{a} \otimes n_{b ; j}$ is used. $I_{a}$ and $I_{b}$ are the identity operators acting on the subsystems (a) and (b), respectively.

Since $\operatorname{Tr}\left\{n_{r ; i}^{+} n_{r ; j}\right\}=\delta_{i j}(r=a, b)$, then $\operatorname{Tr}\left[\left(n_{a ; i} \otimes n_{b ; k}\right)^{+}\left(n_{a ; j} \otimes n_{b ; l}\right)\right]=$ $\operatorname{Tr}\left[n^{+}{ }_{a ; i} n_{a ; j}\right] \operatorname{Tr}\left[n^{+}{ }_{b ; k} n_{b ; l}\right]=\delta_{i j} \delta_{k l}$ and, consequently,

$$
H_{p q}=2 \operatorname{Tr}\left[\left(n_{a ; p} \otimes n_{b ; q}\right)^{+} H_{i n t}\right\rfloor
$$

Under any $\mathbf{S O}(3)$ rotation, $I_{a}$ and $I_{b}$ operators are invariant whereas $\vec{n}_{a}$ and $\vec{n}_{b}$ behave as vectors. Hence, the operators $\vec{n}^{(a)}=\vec{n}_{a} \otimes I_{b}$ and $\vec{n}^{(b)}=I_{a} \otimes \vec{n}_{b}$ also behave as vectors. Then, a second-rank tensor, $\tilde{V}$, can be built whose components in the reference frame, $\sigma \equiv(x$, $y, z)$, are given by (46) and the $H_{\text {int }}$ expansion (45) in any reference frame takes the form:

$$
H_{\text {int }}=\frac{1}{2} \vec{n}^{(a)} \cdot \tilde{V} \cdot \vec{n}^{(b)}
$$

Let us now consider a magnetic field, $\vec{B}$, acting on the system. The Hamiltonian will be:

$$
H=H_{a}+H_{b}+H_{i n t}+H_{a, Z e}+H_{b, Z e}=H_{0}+H_{p}
$$

where the Zeeman interaction for both subsystems, $H_{a, Z e}$ and $H_{b, Z e}$, has been included in $H_{p}$. Considering a weak coupling among the subsystems (a) and (b), we have to describe the perturbation Hamiltonian, $H_{p}$, within the ground quartet. Taking into account (26) and (46) it can be written as a function of $\vec{n}^{(a)}$ and $\vec{n}^{(b)}$ vector operators as:

$$
H_{p}=\mu_{B} \vec{B} \cdot \tilde{g}_{a} \cdot\left(\frac{1}{\sqrt{2}} \vec{n}^{(a)}\right)+\mu_{B} \vec{B} \cdot \tilde{g}_{b} \cdot\left(\frac{1}{\sqrt{2}} \vec{n}^{(b)}\right)+\left(\frac{1}{\sqrt{2}} \vec{n}^{(a)}\right) \cdot \tilde{V} \cdot\left(\frac{1}{\sqrt{2}} \vec{n}^{(b)}\right)
$$

where $\tilde{g}_{a}$ and $\tilde{g}_{b}$ are the gyromagnetic tensors of the systems (a) and (b) as defined in section 3 .

It is important to realize that $H_{p}$ acts as perturbation on the physical states corresponding to the four-fold degenerated ground state of the non-interacting pair. We will to build an fictitious spins Hamiltonian able to reproduce the behaviour of this physical system. How to achieve this will be briefly sketched although some mathematical rigor may be lost. 
The fictitious spin formalism in the case of a Kramer doublet yields the substitution rule where the $\vec{n}$ vector operator should be replaced by a fictitious spin operator, namely $2 \vec{S}$, see (28). If we now introduce the state space of two one-half spins, $\vec{S}_{a}$ and $\vec{S}_{b}$, the following substitution rules can be derived: $\vec{n}^{(a)}=\vec{n}_{a} \otimes I_{b} \rightarrow \sqrt{2} \vec{S}_{a} \otimes I_{b}=\sqrt{2}\left(\vec{S}_{a} \otimes I_{b}\right)$, $\vec{n}^{(b)}=I_{a} \otimes \vec{n}_{b} \rightarrow \quad I_{a} \otimes \sqrt{2} \vec{S}_{b}=\sqrt{2}\left(I_{a} \otimes \vec{S}_{b}\right)$. Then, using the shorthand notation $\left(\vec{S}_{a} \otimes I_{b}\right)=\vec{S}_{b}$ and $\left(I_{a} \otimes \vec{S}_{b}\right)=\vec{S}_{b}$, a (fictitious) spin Hamiltonian, $H^{s}$, equivalent to (49) is given by:

$$
H^{S}=\mu_{B} \vec{B} \cdot \tilde{g}_{1} \cdot \vec{S}_{1}+\mu_{B} \vec{B} \cdot \tilde{g}_{2} \cdot \vec{S}_{2}+\vec{S}_{1} \cdot \tilde{V} \cdot \vec{S}_{2}
$$

where the two first terms account for a Zeeman interaction for the two one-half spins and the last one corresponds to a bilinear coupling between both spins. Note that (50) act on the space of state of two one-half (fictitious) spins $\vec{S}_{a}$ and $\vec{S}_{b}$ while (49) acts on the low energy physical states.

In general $\tilde{V}$ can be decomposed as a sum of its symmetric part, which will be denoted by $\widetilde{K}^{s}$, and its antisymmetric part determined by a vector under $\mathbf{S O}(3), \vec{W}$. Moreover, taking the scalar $K_{0}=(1 / 3) \operatorname{tr}\left(\tilde{K}^{s}\right)$ and the symmetric traceless tensor $\widetilde{K}=\tilde{K}^{S}-K_{0}$, the coupling term in (50) can be written as [B099b]

$$
\vec{S}_{a} \cdot \tilde{V} \cdot \vec{S}_{b}=\vec{S}_{a} \cdot \tilde{K}^{S} \cdot \vec{S}_{b}+\vec{W} \cdot\left(\vec{S}_{a} \wedge \vec{S}_{b}\right)=K_{0}\left(\vec{S}_{a} \cdot \vec{S}_{b}\right)+\vec{S}_{a} \cdot \tilde{K} \cdot \vec{S}_{b}+\vec{W} \cdot\left(\vec{S}_{a} \wedge \vec{S}_{b}\right)
$$

The first contribution in (51), $K_{0}\left(\vec{S}_{a} \cdot \vec{S}_{b}\right)$, is interpreted as an isotropic exchange interaction (Heisenberg interaction) between the (fictitious) spins whereas the second one, $\vec{S}_{a} \cdot \tilde{K} \cdot \vec{S}_{b}$ represents a traceless symmetric exchange interaction, sometimes denoted as a pseudo-dipolar [KA93] or asymmetric [BO99] interaction. The last one, $\vec{W} \cdot\left(\vec{S}_{a} \wedge \vec{S}_{b}\right)$, is identified with the Dzyaloshinkii-Moriya interaction or antisymmetric exchange interaction [BO99].

With the aim of illustrating the formerly presented formalism we will consider a simple (rather academic) example in order to lighten mathematical details. Let two identical rare earth Kramer ions be in an axial local symmetry placed with their distinguished axis $\left(z_{1}\right.$ and $z_{2}$, respectively) rotated, in opposite direction, an angle $\theta$ around a common direction normal to the line joining the two ions (see figure 4). The principal values of the $g$-tensor for 
the ground KD of the ions will be denoted as $g_{\|}$and $g_{\perp}$ and $\left(x_{a}, y_{a}, z_{a}\right)$ and $\left(x_{b}, y_{b}, z_{b}\right)$ are the principal axes of the $g$-tensor of (a) and (b), respectively. All the calculations will be referred to a common reference frame $(X, Y, Z)$ with the $Z$ axis along the direction joining both ion and with the $X$ axis in the common principal direction of both $g$-tensor (see figure 4)

Let $\vec{J}_{r}(r=a, b)$ be the angular momentum operator of each ion (with $J_{a}=J_{b}=J$ ) and $g_{J}$ the corresponding free ion $g$-value. Let us also assume an interaction between both ions given by a Heisenberg term

$$
H_{i n t}=\varepsilon\left(\vec{J}_{a} \cdot \vec{J}_{b}\right)
$$

As an application of the Wigner-Eckart theorem, within any manifold of a given angular momentum $J_{r}(r=\mathrm{a}, \mathrm{b})$, the following equivalence can be used:

$$
\vec{N}_{(r)}=\vec{L}_{r}+g_{e} \vec{S}_{r}=g_{J} \vec{J}_{r} \quad(r=a, b)
$$

Consequently, see (24):

$$
\vec{J}_{r}=\frac{1}{g_{J}} \vec{N}_{(r)}=\frac{1}{g_{J}} \frac{1}{\sqrt{2}} \tilde{g}_{r} \cdot \vec{n}_{r} \quad(r=a, b)
$$

and (52) is given in any reference frame, $\sigma \equiv(x, y, z)$, as

$$
H_{i n t}=\frac{1}{2} \sum_{i, j=x, y, z}\left\{\left(\frac{\varepsilon}{g_{J}^{2}}\right) \sum_{k=x, y, z} g_{a, k i} g_{b, k j}\right\} n_{(a) i} n_{(b) j}
$$

since $\tilde{g}_{r}(r=a, b)$ is a symmetric tensor (see section 3). Then, the matrix expression of $\tilde{V}$, see (47), in any reference frame is given by:

$$
V=\frac{\varepsilon}{g_{j}^{2}}\left(g_{1} \cdot g_{2}\right)
$$

where $g_{r}(r=a, b)$ is the matrix expression of $\tilde{g}_{r}$ in this reference frame. In particular, referring to the reference frame $(X, Y, Z)$ above defined, see figure 4 , as

$$
g_{r}=\left(\begin{array}{ccc}
g_{\perp} & 0 & 0 \\
0 & g_{\perp} \cos ^{2} \theta+g_{\|} \sin ^{2} \theta & \mp\left(g_{\|}-g_{\perp}\right) \sin \theta \cos \theta \\
0 & \mp\left(g_{\|}-g_{\perp}\right) \sin \theta \cos \theta & g_{\perp} \sin ^{2} \theta+g_{\|} \cos ^{2} \theta
\end{array}\right)
$$


where the sign minus (plus) in the off-diagonal non-null element corresponds to $r=a(r=b)$. Then:

$$
V=\frac{\varepsilon}{g_{J}^{2}}\left(\begin{array}{ccc}
g_{\perp}^{2} & 0 & 0 \\
0 & \frac{g_{\|}^{2}+g_{\perp}^{2}}{2}-\frac{g_{\|}^{2}-g_{\perp}^{2}}{2} \cos 2 \theta & \left(\frac{g_{\|}-g_{\perp}}{2}\right)^{2} \sin 4 \theta \\
0 & -\left(\frac{g_{\|}-g_{\perp}}{2}\right)^{2} \sin 4 \theta & \frac{g_{\|}^{2}+g_{\perp}^{2}}{2}+\frac{g_{\|}^{2}-g_{\perp}^{2}}{2} \cos 2 \theta
\end{array}\right)
$$

Hence, we obtain

$$
\begin{gathered}
K_{0}=\left(\frac{g_{\|}^{2}+2 g_{\perp}^{2}}{3 g_{J}^{2}}\right) \varepsilon \\
K=\left(\frac{g_{\|}^{2}-g_{\perp}^{2}}{3 g_{J}^{2}}\right) \varepsilon\left(\begin{array}{ccc}
-1 & 0 & 0 \\
0 & 3 \sin ^{2} \theta-1 & 0 \\
0 & 0 & 3 \cos ^{2} \theta-1
\end{array}\right)
\end{gathered}
$$

Thus, the principal axes of the effective traceless symmetric exchange interaction coincide with the axes of the $(X, Y, Z)$ reference frame . Finally, the components of the $\vec{W}$ vector, which define the Dzyaloshinkii-Moriya interaction, are:

$$
W_{X}=\left(\frac{g_{\|}-g_{\perp}}{2 g_{J}}\right)^{2} a \sin 4 \theta \quad W_{Y}=0 \quad W_{Z}=0
$$

Note that the symmetric exchange interaction appears as a consequence of the anisotropy of the individual $g$-tensors whereas, the antisymmetric interaction results from the non-coaxial arrangement of their principal axes.

\section{CONCLUDING REMARKS}

It is well-known that the paramagnetic behaviour of a $\mathrm{KD}$ can be experimentally described as an $S=1 / 2$ entity characterized by a $g$-tensor. A complete set of EPR experiments determines the absolute values of its principal values, their principal directions and the sign of the product of the three principal values. However, at present there is no clear way to compare the theoretical predictions with the above mentioned experimental information in a general case since some ambiguities appear. 
In this work, a procedure to extract all this experimentally obtained information has been established when the electronic structure of the KD is known, regardless the level of the used theory. This procedure also gives precise rules to build the suitable spin Hamiltonian by the substitution of some physical operators (the magnetic moment components in a suitable reference frame) with the corresponding spin operators. In this way, a univocal way to compare the information coming from paramagnetic resonance experiments with the predictions derived from a microscopic model of the paramagnetic Kramers entity is provided for a ground state being a well isolated KD.

Those substitution rules also provide a way to build a fictitious spin Hamiltonian to account for the magnetic behaviour of the lowest energy levels of a pair of weakly interacting Kramers systems. This fictitious spin Hamiltonian, expressed in a multispin scheme, include the Zeeman interaction of two isolated one half fictitious spins (described by the corresponding $g$-tensors) and an (effective) exchange interaction between both spins. This exchange interaction includes isotropic, symmetric traceless and antisymetric terms. Explicit relations between the parameters of that effective exchange interaction and those describing the physical interaction are given. On the basis of these results, further extensions for describing more complex systems of several interacting Kramers moieties may be developed.

\section{ACKNOWLEDGEMENTS.}

This work was supported by Spanish Ministry of Economy and Competitively (MINECO), projects $\mathrm{n}^{\circ}$ MAT2011-23861 to JIM and by “Grupos de investigación” Program of the Aragon Autonomous Government, refs. B18 and E33.

\section{Appendix A.}

Each a $m \times n$ real matrix $A$ (with $m \geq n$ ) can be factorized as $A=U \cdot W \cdot V$, where $U$ is a $m \times n$ real matrix fulfilling $U^{t} U=I_{n}$, the $n \times n$ identity matrix, $V$ is a $n \times n$ orthogonal matrix, $V \in$ $\mathbf{O}(\mathbf{n})$, and $W$ is a $n \times n$ diagonal real matrix with all its diagonal elements non-negative [PT92, DE97]. The diagonal elements of $W$ are the singular values of $A$ and the factorization $A=$ $U \cdot W \cdot V$ is called the singular value decomposition (SVD) of the matrix $A$.

If $A$ is a square matrix of dimension $n$ then $U \in \mathbf{O}(\mathbf{n})$ as well. Any element of $A$ is given by $A_{i j}=\Sigma_{k=1, n}\left(w_{k} U_{i k} V_{j k}\right)$, where $w_{k}$ stands for the diagonal elements of $W$, the singular 
values of $A$. Consequently, as the change of the sign of the elements of a column of a square matrix induces a change of the sign of its determinant, if the restriction $w_{k} \geq 0$ is lifted, it is possible to take $U$ and $V$ as direct rotation, $U, V \in \mathbf{S O ( n ) . ~ T h i s ~ r e s u l t ~ h a s ~ b e e n ~ u s e d ~ i n ~}$ deriving equation (13).

\section{APPENDiX B.}

First definitions and nomenclature in group theory can be obtained, for instance, in [HE60, HA62, TI64b, CO90] and a more depth discussion on the structure of groups and on the homomorphism decomposition, as it is used in the next paragraph, can be read in [HU74].

Let $\mathbf{G}$ be a point symmetry group (PSG). $\mathbf{G}$ is a subgroup of the full three dimensional orthogonal group, $\mathbf{O}(3)$. $\mathbf{S O}(3)$ is the special orthogonal group: its elements are the direct rotations belonging to $\mathbf{O}(3)$. So, $\mathbf{S O}(3)$ is given by those $\mathbf{O ( 3 )}$ transformations whose determinants are equal to one. Lastly, $\mathbf{C}_{\mathbf{i}}$ denotes the point group formed by the identity, $I$, and the origin inversion, $J_{O}$. Both, $\mathbf{S O}(3)$ and $\mathbf{C}_{\mathbf{i}}$, are normal subgroups of $\mathbf{O}(3)$ and $\mathbf{O}(3)=$ $\mathbf{S O}(3) \otimes \mathbf{C}_{\mathbf{i}}$. So, any transformation $R \in \mathbf{O}(3)$ either belongs to $\mathbf{S O}(3)$ or there exists an unique $Q$ (in fact, $Q=R \cdot J_{O}$ ) belonging to $\mathbf{S O}(3)$ and accomplishing $R=Q \cdot J_{O}=J_{O} \cdot Q$. The projection, $\pi$, of $\mathbf{O}(3)$ onto $\mathbf{S O}(3)$, that verifies $\pi(R)=R$ if $R \in \mathbf{S O}(3)$ and $\pi(R)=R \cdot J_{O}$ otherwise, is a group homomorphism. Its kernel is $\mathbf{C}_{\mathbf{i}}, \operatorname{Ker}(\pi)=\mathbf{C}_{\mathbf{i}}$. On the other hand, the inclusion, $\chi$, of $\mathbf{G}$ to $\mathbf{O}(3)$, such that $\chi(T)=T$ for any operation of $\mathbf{G}$, is also a group homomorphism. Consequently, the application $\zeta=\pi^{\circ} \chi$ is an homomorphism of $\mathbf{G}$ in $\mathbf{S O}(3)$. Its image is a subgroup of $\mathbf{S O}(3)$ that will be denoted as $\hat{\mathbf{G}}$. Besides $\operatorname{Ker}(\zeta)=\mathbf{G} \cap \mathbf{C}_{\mathbf{i}}$. So, $\zeta$ is injective if and only if the inversion does not belong to $\mathbf{G}$. In a similar way, given a point symmetry group, $\mathbf{G}$, all direct transformation in $\mathbf{G}$ form a subgroup of it, which will be denoted as $\mathbf{S G}$. Then, $\mathbf{S G}=$ $\mathbf{G} \cap \mathbf{S O}(3)$. The SG subgroup of the relevant PSG in Chemistry [CO90] are collected in table 1 .

Table 1. Classification of the PSG relevant in chemistry in cases I, II and III indicating the corresponding SG and $\hat{\mathbf{G}}$ groups.

\begin{tabular}{cccc}
\hline G & SG & case & $\hat{\mathbf{G}}$ \\
\hline $\mathrm{C}_{\mathrm{i}}$ & $\mathrm{C}_{1}$ & $\mathrm{II}$ & $\mathrm{C}_{1}$ \\
$\mathrm{C}_{\mathrm{s}}$ & $\mathrm{C}_{1}$ & $\mathrm{III}$ & $\mathrm{C}_{2}$
\end{tabular}




\begin{tabular}{ccccc}
$\mathrm{C}_{\mathrm{n}}$ & & $\mathrm{C}_{\mathrm{n}}$ & $\mathrm{I}$ & $\mathrm{C}_{\mathrm{n}}$ \\
$\mathrm{D}_{\mathrm{n}}$ & & $\mathrm{D}_{\mathrm{n}}$ & $\mathrm{I}$ & $\mathrm{D}_{\mathrm{n}}$ \\
$\mathrm{C}_{\mathrm{nv}}$ & & $\mathrm{C}_{\mathrm{n}}$ & $\mathrm{III}$ & $\mathrm{D}_{\mathrm{n}}$ \\
$\mathrm{C}_{\mathrm{nh}}$ & (n even) & $\mathrm{C}_{\mathrm{n}}$ & $\mathrm{II}$ & $\mathrm{C}_{\mathrm{n}}$ \\
& (n odd) & $\mathrm{C}_{\mathrm{n}}$ & $\mathrm{III}$ & $\mathrm{C}_{2 \mathrm{n}}$ \\
$\mathrm{D}_{\mathrm{nh}}$ & (n even) & $\mathrm{D}_{\mathrm{n}}$ & $\mathrm{II}$ & $\mathrm{D}_{\mathrm{n}}$ \\
& (n odd) & $\mathrm{D}_{\mathrm{n}}$ & $\mathrm{III}$ & $\mathrm{D}_{2 \mathrm{n}}$ \\
$\mathrm{D}_{\mathrm{nd}}$ & (n even) & $\mathrm{D}_{\mathrm{n}}$ & $\mathrm{III}$ & $\mathrm{D}_{2 \mathrm{n}}$ \\
& (n odd) & $\mathrm{D}_{\mathrm{n}}$ & $\mathrm{II}$ & $\mathrm{D}_{\mathrm{n}}$ \\
$\mathrm{S}_{2 \mathrm{n}}$ & (n even) & $\mathrm{C}_{\mathrm{n}}$ & $\mathrm{III}$ & $\mathrm{C}_{2 \mathrm{n}}$ \\
& (n odd) & $\mathrm{C}_{\mathrm{n}}$ & $\mathrm{II}$ & $\mathrm{C}_{\mathrm{n}}$ \\
$\mathrm{T}$ & & $\mathrm{T}$ & $\mathrm{I}$ & $\mathrm{T}$ \\
$\mathrm{T}_{\mathrm{h}}$ & & $\mathrm{T}$ & $\mathrm{II}$ & $\mathrm{O}$ \\
$\mathrm{T}_{\mathrm{d}}$ & & $\mathrm{T}$ & $\mathrm{III}$ & $\mathrm{O}$ \\
$\mathrm{O}$ & & $\mathrm{O}$ & $\mathrm{I}$ & $\mathrm{O}$ \\
$\mathrm{O}_{\mathrm{h}}$ & & $\mathrm{O}$ & $\mathrm{II}$ & $\mathrm{O}$ \\
\hline
\end{tabular}

If $\mathbf{G}$ only contains direct rotations, $\mathbf{S G}=\mathbf{G}$, then $\mathbf{G}$ is a subgroup of $\mathbf{S O}(3)$ and $\zeta$ is the inclusion, consequently $\hat{\mathbf{G}}=\mathbf{G}$ (case I). In the opposite case there is an improper operation, $R$, in $\mathbf{G}$ and two possibilities appear. If $R \cdot J_{O}$ is also in $\mathbf{G}$ then $\mathbf{C}_{\mathbf{i}}$ is a subgroup; so, $\mathbf{G}=\mathbf{S G} \otimes \mathbf{C}_{\mathbf{i}}$ and then, $\hat{\mathbf{G}}=\mathbf{S G}$ (case II). Otherwise, $\hat{\mathbf{G}}$ is isomorphic to $\mathbf{G}$ but both, $\hat{\mathbf{G}}$ and $\mathbf{G}$, are different (case III). $\mathbf{C}_{\mathbf{n}}$ is an example of case I, $\mathbf{O}_{\mathbf{h}}$ of case II and $\mathbf{T}_{\mathbf{d}}$ of case III. In the last two cases $\hat{\mathbf{G}}$ $=\mathbf{O}$, the group of direct rotations of the octahedron. Table 1 also shows the classification of all PSG in those three cases and the corresponding $\hat{\mathbf{G}}$ PSG. In summary, if $\breve{\mathbf{G}}$ is the group product of $\mathbf{G}$ and $\mathbf{C}_{\mathbf{i}}$, a subgroup of $\mathbf{O}(\mathbf{3})$, then $\hat{\mathbf{G}}=\mathbf{S} \breve{\mathbf{G}}$. In the practice the elements of $\hat{\mathbf{G}}$ are all direct rotations in $\mathbf{G}$ and those built as product of any element of $\mathbf{G}$ and the origin inversion.

Let us consider a paramagnetic entity whose magnetic behaviour is described by (1). If $R$ is a transformation belonging to its symmetry group, $\mathbf{G}$, the magnetic response of the system should be the same for a magnetic field $\vec{B}$ and for the transformed magnetic field $R(\vec{B})$. On the other hand, since $\vec{B}$ is a vector that remains invariant under the origin inversion, it turns out that the magnetic response of the system should be the same if $R$ is any element of $\breve{\mathbf{G}}$. Also, the invariance of $\vec{B}$ under the origin inversion allows us to consider only 
those transformation operations in $\hat{\mathbf{G}}$. Hence $\hat{\mathbf{G}}$ results to be the relevant PSG for analysing the magnetic properties of our system.

A classification of the crystallographic PSG in eleven "aggregates" was given by Roitsin [RO81]. The present construction of $\hat{\mathbf{G}}$ is supported by the same arguments than those given there for the "aggregates" classification of the PSG although our objective is more modest. While [RO81] pursues the classification of a "Generalized Spin Hamiltonian" of any paramagnetic entity based on symmetry arguments we are dealing here with the simpler case of a $\mathrm{KD}$ whose $\mathrm{SH}$, as shown in section 3, is characterized by a $g$-tensor and our goal is to prove that with our construction the $g$-tensor should reflect the point symmetry of the Kramer entity. However, note that the $\hat{\mathbf{G}}$ group is a suitable label for the Roitsin's aggregates and this group is the adequated one to describe the symmetry properties of the $\mathrm{SH}$. 


\section{REFERENCES}

AA09 P.J. Alonso, A.B. Arauzo, M.A. García-Monforte, A. Martín, B. Menjón, C. Rillo, M. Tomás. Homoleptic organoderivatives of high-valence Nickel(III). Chem. Eur. J. 15 (2009) 11020-11030 (doi: 10.1002/chem.200901259).

AB70a A. Abragam, B. Bleaney, Electron Paramagnetic Resonance of Transitions Ions, Oxford University Press, Oxford, 1970. Chapter 15.

AB70b A. Abragam, B. Bleaney, Electron Paramagnetic Resonance of Transitions Ions, Oxford University Press, Oxford, 1970. Chapter 3.

AB70c A. Abragam, B. Bleaney, Electron Paramagnetic Resonance of Transitions Ions, Oxford University Press, Oxford, 1970. Chapter 9.

AB70d A. Abragam, B. Bleaney, Electron Paramagnetic Resonance of Transitions Ions, Oxford University Press, Oxford, 1970. Chapter 5.

AL15 G. Aromí, F. Luis, O. Roubeau. Lanthanides complexes as realizations of Qubits and Qugates for quantum computing. in lathanides and actinides in molecular magnetism, (R.A. Layfield, M. Muegesu, ed). Wiley-VCH, Weinheim, Gernmany, 2015. Ch- 7. (ISBN 978-3-527-33526-8).

AM07 P.J. Alonso, J.I. Martínez, I. García-Rubio, The study of the ground state Kramers doublet of low heminic system revisited. A comprehensive description of the EPR and Mössbauer spectra, Coord. Chem. Rev. 251 (2007) 12-24. (doi: 10.1016/j.ccr.2006.05.007).

AP51 A. Abragam, M.H.L. Pryce, Theory of nuclear hyperfine structure of paramagnetic resonance spectra in crystrals, Proc. Roy. Soc. 205 (1951) 135153. (doi: 10.1098/rspa.1951.0022)

AS71 C.H. Anderson, E.S. Sabisky. WPR studies of Photocrhomic CAF2. Phys. Rev. 3 (1971) 527-536. (doi: 10.1103/PhysRevB.3.527).

BB56 B. Bleaney, M.C.M. O’Brian, paramagnetic resonance of some complex cyanides of the iorn group. II theory, Proc. Phys. Soc. B69, (1956) 1216-1230. (doi: 10.1088/0370-1301/69/12/305).

BB82 M. Barzaghi, T. Beringhelli, F. Morazzoni, Oxygen chemisorption on $\gamma-\mathrm{Al}_{2} \mathrm{O}_{3}$ phthalocyaninato-cobalt(II): coordination of $\mathrm{O}_{2}$ by complexed cobalt centers; tumbling of molecular oxygen on surface cobalt centers, J. Mol. Catal. 14 (1982) 357-374. (doi: 10.1016/0304-5102(82)80096-5)

BC73 C. Busetto, F. Cariati, A. Fusi, M. Gullotti, F. Morazzoni, A. Pasini, R. Ugo, Optically active complexes of Schiff bases. Part II. Complexes of cobalt(II) with tetradentate Schiff bases and their reactivity with oxygen, J. Chem. Soc., Dalton Trans. (1973) 754-765. (doi: 10.1039/dt9730000754).

BG90 A. Bencini, D. Gatteschi, EPR of Exchange Coupled Systems, Springer, Berlin, 1990.

BG09 M. Brustolon, E. Giamello. Electron paramagnetic Resonance: A Practitioner’s Toolkit. John-Wiley and Sons Hoboken, NJ, USA, 2009. 
(ISBN: 978-0471-75496-1)

BM70 R.B. Bentley, F.E. Mabbs, W.R. Smail, M. Gerloch, J. Lewis, Electron spin resonance and bulk paramagnetism studies of some diarylbis(diethylphenylphosphine)cobalt(II) complexes, J. Chem. Soc. A (1970) 3003-3009. (doi: 10.1039/j19700003003).

BO77 T.L. Bohan, Analysis of low-spin ESR spectra of ferric hemeproteins: a reexamination, J. Magn. Reson. 26 (1977) 109-118. (doi: 10.1016/00222364(77)90240-2).

BO99 R. Boča, Theoretical Foundations of Molecular Magnetism, Elsevier, Amsterdam, 1999. Chapter 10.

BO99b R. Boča, Theoretical Foundations of Molecular Magnetism, Elsevier, Amsterdam, 1999. Chapter 1.

BO03 H. Bolvin. From ab initio calculations in model Hamiltonians; The effective Hamiltonian technique as an efficient tool to describe mixed-valence molecules. J. Pys. Chem. A 107 (2003) 5071-5078 (doi: 10.1021/jp034176).

BO06 H. Bolvin. An alternative approach to the g-matrix: theory and applications. ChemPhysChem 7, 1575-1589 (2006). (doi: 10.1002/cphc.200600051)

BS03 R. Benda, V. Schünemann, A.X. Trautwein, S. Cai, J.R. Polam, C.T. Watson, T.K. Shikhireva, F.A. Walker, Models of bis-histidine-coordinated ferricytochromes: Mössbauer and EPR spectroscopic studies of low-spin iron(III) tetrapyrroles of various electronic ground states and axial ligand orientations, J. Biol. Inorg. Chem. 8 (2003) 787-801. (doi: 10.1007/s00775003-0472-5).

CA72 M.J. Caola, The spin Hamiltonian, J. Phys. A: Gen. Phys. 5 (1972) 16491657. (doi: 10.1088/0305-4470/5/12/003).

CA86 R.L. Carlin, Magnetochemistry, Springer, Berlin, 1986. Chapter 5.

CC08 L.F. Chiboratu, A. Ceulemans, H. Bolvin, Unique definition of the Zeemansplitting g-tensor of a Kramers doublet, Phys. Rev. Lett. 101 (2008) 033003. (doi: 10.1103/PhysRevLett.101.033003).

CO90 F.A. Cotton. Chemical Applications of Group Theory, 3rd ed. Wiley (NY, 1990) (ISBN 978-0-471-51094-9)

CU12 L.F. Chiboratu, L. Ungur, Ab initio calculation of anisotropic magnetic properties of complexes. I. Unique definition of pseudospin Hamiltonians and their derivation, J. Chem. Phys. 137 (2012) 064112. (doi: 10.1063/1.4739763).

DE97 J.W. Demmel, Applied Numerical Linear Algebra, SIAM, Society for Industrial and Applied Mathematics, Philadelphia, 1997. Chapter 3.

DU93 D.E. Dugdale, The theoretical basis of spin Hamiltonian theory, J. Phys.: Condens. Matter. 5 (1993) 7837-7846. (doi: 10.1088/0953-8984/5/42/006). 
EL53 R.J. Elliott, Crystal field theory in rare earths, Rev. Mod. Phys. 25 (1953) 167-169. (doi: 10.1103/RevModPhys.25.167).

ES51 R.J. Elliott, K.W.H. Stevens, A preliminary survey of the paramagnetic resonance phenomena observed in rare earth ethyl sulphates, Proc. Phys. Soc. A64 (1951) 205-207. (doi: 10.1088/0370-1298/64/2/115).

ES52 R.J. Elliott, K.W.H. Stevens, the theory of the magnetic properties of rare earth salts: cerium ethyl sulphate, Proc. Phys. Soc. A215 (1952) 437-453. (doi: 10.1098/rspa.1952.0223).

ES53 R.J. Elliott, K.W.H. Stevens, The theory of magnetic resonance experiments on salts of the rare earths, Proc. Phys. Soc. A218 (1953) 553-566. (doi: 10.1098/rspa.1953.0124).

F067 M.E. Foglio, The determination of the sign of the spin-Hamiltonian parameters, Nuovo Cimento 50 (1967) 158-160. (doi: 10.1007/BF02710690).

GA75 B.R. McGarvey, Theory of the spin Hamiltonian parameters for low spin Co(II) complexes, Can J. Chem. 53 (1975) 2498-2511. (doi: 10.1139/v75355).

GA98a B.R. McGarvey, Survey of ligand field parameters of strong field $\mathrm{d}^{5}$ complexes obtained from the g matrix, Coord. Chem. Rev. 170 7(1998) 5-92. (doi: 10.1016/S0010-8545(97)00073-8).

GA98b B.R. McGarvey, The ESR g matrix for strong field $\mathrm{d}^{5}$ complexes, Quim, Nova. 21 (1998) 206-213. (doi; 10.1590/S0100-40421998000200017).

GA14 M.A. García-Monforte, I. Ara, A. Martín, B. Menjón, M. Tomás, P.J. Alonso, A.B. Arauzo, J.I. Martínez, C. Rillo. Homoleptic organocobalt(III) compounds with intermediate spin. Inorg. Chem. 53 (2014) 12384-12395. (doi: 10.1021/ic501719y).

GG06 O. Guillot-Nöel, Ph. Goldner, Y. Le Du, E Baldit, P. Monnier, K. Bencheikh. Hyperfine interaction of $\mathrm{Er}^{3+}$ ions in $\mathrm{Y}_{2} \mathrm{SiO}_{5}$ : an electron paramagnetic resonance study. Phys. Rev. B 74 (2006) 214409 (doi: 10.1103/ PhysRevB.74.214409)

GI57 J.F. Gibson, D.J.E. Ingram, Binding in Haemoglobin azide as determined by electron resonance, Nature 180 (1957) 29-30. (doi: 10.1038/180029a0).

GK02 K.J. Guedes, K. Krambrock, J.Y. Gesland. Identification of trivalent rare earth impurities in $\mathrm{YF}_{3}, \mathrm{LuF}_{3}$ and $\mathrm{LiYF}_{4}$ by electron paramagnetic resonance. $\mathrm{J}$. Alloys Compd. 244 (2002) 251-254. (doi: 10.1016/S0925-8388(02)00362-6).

GM75 M. Gerloch, R.F. McMeeking. Paramagnetic properties of unsymmetrical transition-metal complexes. J. Chem. Soc.; Dalton trans. 2443-2451 (1975). (doi: 10.1039/dt9750002443).

GN13 D. Ganyushin, F. Neese. A fully variational spin-orbit coupled complete active space self-consistent field approach: Application to electron paramagnetic resonance g-tensors. J. Chem. Phys., 138, 104113 (2013) (doi: 
10.1063/1.4793736).

GR57 J.S. Griffith, Theory of electron resonance in ferrihaemo-globin azide, Nature 180 (1957) 30-31. (doi: 10.1038/180030a0).

GR58 J.S. Griffith, The electronic structures of some first transition series metal phorphyrins and phthalocyanines, Discuss. Faraday Soc. 26 (1958) 81-86. (doi: 10.1039/DF9582600081).

GR67 J.S. Griffith, Transformations of a spin-Hamiltonian induced by reassignments of fictitious spin states, Molec. Phys. 12 (1967) 359-363. (doi 10.1080/00268976700101591).

HA62 M. Hamermesh. Group Theory and its Application to Physical problems. Adisson-Wesley, Reading, USA. 1962. (ISBN 0201027801)

HE60 W Heine. Group Theory in Quantum Mechanism. Pergamon Press, Oxford, 1960 (ISBN 08-009242-X)

HU74 T.W. Hungerford. Algebra. Holt, Rinehart and Winton Inc. (NY, 1974). Ch 2

JE82 J. Jeener, Superoperators in magnetic resonance, Adv. Magn. Reson. 10 (1982) 1-51. (ISBN 0-12-025510-3)

KA85 O. Kahn, Orbital mechanism of the exchange interaction, in: R.D. Willet, D. Gatteschi, O. Kahn (Eds.), Magneto-Structural Correlations in Exchange Coupled Systems, NATO ASI Series, Series C, vol 140. Kluwer Academic Published, Dordrecht, Holland, 1985. Pages 37-56.

KA93 O. Kahn, Molecular Magnetism, VCH Published Inc., NY, 1993.

KB04 M. Kaupp, M. Bühl, V.G. Malkin (Eds.), Calculation of NMR and EPR Parameters, Wiley-VCH, Wienheim, 2004.

KE02 A.J. Kenyon. Recent develpments in rare-earth doped material for optoelectronics. Prog. Quantum Electron. 26 (2002) 225-284 (doi: 0.1016/S0079-6727(02)00014-9)

LO60 W. Low, Paramagnetic resonance in solids, Solid State Physics Supl. 2, Academic Press, N.Y., 1960.

LS12 J. Luzón, E. Sessoli. Lanthanides in molecular magnetism: so fascinating, so challenging. Dalton trans. 41 (2012) 13556-13567 (doi: 10-1039/c2dt31388j).

MC97 S.K. Misra, Y. Chang, J. Felsteiner. A calculation of the effective g-tensor values for the $\mathrm{R}^{3+}$ ions in $\mathrm{RBa}_{2} \mathrm{Cu}_{3} \mathrm{O}_{7-\delta}$ and $(\mathrm{R}=$ rare earth): low temperature ordering of rare earth moments. J. Phys. Chem. Sol. 58 (1997) 1-11. (doi: 10.1016/S0022-3697(96)00110-2).

MC14 J.P. Malrieu, R. Caballol, C.J. Calzado, C. de Graaf, N. Guihéry, magnetic interactions in molecules and highly correlated materials: physical content, analytical derivation and rigorous extraction of magnetic Hamiltonians, Chem Rev. 114 (2014) 429-492. (doi: 10.1021/cr300500z). 
ME64a A. Messiah, Mécanique Quantique, Dunod, Paris, 1964. Part I, page 272.

ME64b A. Messiah, Mécanique Quantique, Dunod, Paris, 1964. Part II, page 572.

ME64c A. Messiah, Mécanique Quantique, Dunod, Paris, 1964. Part I, page 244.

MG10a R. Maurice, C. de Graff, N. Guihéry, Magnetic anisotropy in binuclear complexes in the weak-exchange limit: from multispin to giant-spin Hamiltonian, Phys. Rev. B81 (2010) 214427. (doi:

10.1103/PhysRevB.81.214427)

MG10b R. Maurice, N. Guihéry, R. Bastardis, C. de Graff, Rigorous extraction of the anisotropic multispin Hamiltonian in bimetallic complexes from the exact electronic Hamiltonian, J. Chem. Theory Comput. 6 (2010) 55-65. (doi: 10.1021/ct900473u).

MG13 R. Maurice, C. de Graff, N. Guihéry, Theoretical determination of spin Hamiltonians with isotropic and anisotropic magnetic interactions in transition metal and lanthanide complexes, Phys. Chem. Chem. Phys. 15 (2013) 18784-18804. (doi: 10.1039/c3cp52521j)

MI1 S.K. Misra, Spin Hamiltonian and Site Symmetries for Transition Ion, in: S.K. Misra (Ed.), Multifrequency Electron Paramagnetic Resonance: Theory and Applications, Wiley-VCH, Weinheim, 2011. Chapter 7.

ML09 P. Molina, H. Loro, S. Álvarez-García, L.E. Bausá, E. Martín.Rodríguez, O. Guillot-Noël, Ph. Goldner, M. Bettinelli, P. Ghigna, J. García-Solé. Phys. Rev. B 80 (2009) 054111 (doi: 10.1103/PhysRevB.80.054111).

MP10 R. Maurice, A.M. Pradipto, N. Guihéry, R. Broer, C. de Graff, Antisymmetric magnetic interactions in oxo-bridged copper(II) bimetallic systems, J. Chem. Theory Comput. 6 (2010) 3092-3101. (doi: 10.1021/ct100329n).

MR80 V.V. Minin, Yu.V. Rakitin, V.V. Volkov, G.M. Larin, Influence of conjugated $\pi$ bonds in equatorial ligands on electron structure of low-spin complexes of Co(II), Bull. Acad. Scienc, USSR, Chem. Div. 29, (1980) 15261533. (doi: 10.1007/BF00951208).

NE07 F. Neese, Quantum chemical approaches to Spin Hamiltonian Parameters, in: B.C. Gilbert, M.J. Davies, D.M. Murphy (Eds.), Electron Paramagnetic Resonance, volume 20. RSC, 2007. Pages 73-95.

NE11 F. Neese, First principles approach to spin-Hamiltonian parameters, in: S.K. Misra (Ed.), Multifrequency Electron Paramagnetic Resonance: Theory and Applications, Wiley-VCH, Weinheim, 2011. Chapter 6.

NK78 Y. Nishida, S. Kida, Ground state of the square planar cobalt(II) complexes, Bull. Chem. Soc. Jpn. 51 (1978) 143-149. (doi: 10.1246/bcsj.51.143).

OH72 J. Owen, E.A. Harris, Pair Spectra and Exchange Interactions, in: S. Geschwind (Ed.), Electron Paramagnetic Resonance, Plenum Press, NY, 1972. Pages. 427-492.

PE73 G.E. Pake, T.L. Estle, The Physical Principles of Electron Paramagnetic resonance. $2^{\text {nd }}$ edition, W.A. Benjamin, Inc. Reading, Massachusetts, U.s.A. 
1973 (ISNB:0-8053-7703-4).

PF72 C.P. Poole, Jr., H. Farach, Theory of Magnetic Resonance, WileyInterscience, N.Y., 1972. Chapter 6.

PO83 C.P. Poole, Jr., Electron Spin Resonance. A comprehensive Treatise on Experimental Techniques. $2^{\text {nd }}$ edition, Wiley-Interscience, N.Y, 1983. Page 189 and ff.

PR50 M.H.L. Pryce, A modified perturbation procedure for a problem in paramagnetism, Proc. Phys. Soc. A63 (1950) 25-29. (doi: 10.1088/03701298/63/1/304).

PR59 M.H.L. Pryce, Sign of g in magnetic resonance and the sign of the quadrupole moment of $\mathrm{Np}^{237}$, Phys. Rev. Lett. 3 (1959) 375-375. (doi:

10.1103/PhysRevLett.3.375).

PT92 W.H. Press, S.A. Teukolsky, W.T. Vetterling, B.P. Flannery, Numerical Recipes. The Art of Scientific Computing, $2^{\text {nd }}$ ed., Cambridge University Press, NY, 1992. Chapter 2.

PT03 A.V. Palii, B.S. Tsukerblat, E. Coronado, J.M. Clemente-Juan, J.J. BorrásAlmenar, Microscopic approach to the pseudo-spin-1/2 Hamiltonian for Kramers doublets in exchange coupled Co(II) pairs, Inorg. Chem. 42 (2003) 2455-2458. (doi: 10.1021/ic0259686)

PT10 A.V. Palii, B.S. Tsukerblat, .J.M. Clemente-Juan, E. Coronado, Magnetic exchange between metal ions with unquenched orbital angular moment: basic concepts and relevance to molecular magnetism, Int. Rev. Phys. Chem. 29 (2010) 135-230. (doi: 10.1080/01442350903435256)

PT11 A.V. Palii, B.S. Tsukerblat, S. Klokishner, K.R. Dunbar, J.M. Clemente-Juan, E. Coronado, Beyond the spin model: exchange coupling in molecular magnets with unquenched orbital angular momenta, Chem. Soc. Rev. 40 (2011) 3130-3156. (doi: 10.1039/c0cs00175a)

RI94 H.R. Rieger, Electron paramagnetic resonance studies of low-spin d $\mathrm{d}^{5}$ transition metal complexes, Coord. Chem. Rev. 135/136 (1994) 203-286. (doi: 10.1016/0010-8545(94)80069-3).

RK14 C. Rudowicz, M. Karbowiak, terminological confusion and problems at the interface between the crystal field Hamiltonian and zero-field splitting Hamiltonian - survey of the CF = ZFS confusion in the recent literature, Physica B, 451, 134-150 (2014). (doi: 10.1016/j.physb.2014.06.018).

RK15a C. Rudowicz, M. Karbowiak, Revealing the consequences and errors of substance arising from the inverse confusion between the crystal (ligand) field quantities and the zero-field ones, Physica B, 456, (2015) 330-338. (doi: 10.1016/j.physb.2014.09.011).

RK15b C. Rudowicz, M. Karbowiak. Disentangling intricate web of interrelated notions at the interface between the physical (crystal field) Hamiltonians and the effective (spin) Hamiltonians. Coord. Chem. Rev. 187 (2015) 28-63. (doi: 
10.1016/j.ccr.2014.12.006).

RO88 A. Rockenbauer, Invariance and the uncertainty principle in EPR spectroscopy of crystals and liquids, Compt. Math. Applic. 16 (1988) 629635. (doi: 10.1016/0898-1221(88)90251-9).

RM01 C. Rudowicz, S.K. Misra, Spin-Hamiltonian formalism in electron magnetic resonance (EMR) and related spectroscopies, Appl. Spectr. Rev. 36 (2001) 11-63. (doi: 10.1081/ASR-100103089).

RM14 R. Ruamps, R. Maurice, C. de Graaf, N. Guihéry, Interplay between local anisotropies in binuclear complexes, Inorg. Chem. 53 (2014) 45084516. (doi: 10.10217ic500180k).

R081 A.B. Roitsin. Generalized Spin-Hamiltonian and Low Symmetry Effects in Paramagnetic Resonance. phys. sat. sol. (b) 104 (1981) 11-35. (doi: 10.1002/pssb.2221040102)

RS76 A. Rockenbauer, P. Simon, Ambiguity problems of the spin Hamiltonian, J. Magn. Reson. 22 (1976) 243-263. (doi: 10.1016/0022-2364(76)90299-7).

RS01 C. Rudowicz, H.W.F. Sung, Can the electron magnetic resonance (EMR) techniques measure the crystal (ligand) field parameters?, Physica B, 300 (2001) 1-26. (doi: 10.1016/S0921-4526(01)00568-3).

RU70a R.S. Rubins. Mean g values of rare-earth ions in crystals. Phys. Rev. B 1 (1970) 139-141. (doi: 10.1103/PhysRevB.1.139).

RU70b R.S. Rubins. Mean g values of rare-earth ions: extension to monoclinic symmetry. . Phys. Rev. B 2 (1970) 2813-2816. (doi: 10.1103/PhysRevB.2.2813).

RU87 C. Rudowicz. Concept of spin Hamiltonian, form of zero-field splitting and electronic Zeeman Hamiltonians and relations between parameters used in EPR. A critical review. Magn. Reson. Rev. 13, (1987) 1-89.

RU08a C. Rudowicz, Clarification of the confusion concerning the crystal field quantities vs. the zero-field splitting quantities in magnetism studies: part I survey of literature dealing with specific compounds, Physica B, 403, (2008) 1882-1897. (doi: 10.1016/j.physb.2007.10.219).

RU08b C. Rudowicz, Clarification of the confusion concerning the crystal field quantities vs. the zero-field splitting quantities in magnetism studies: part II survey of literature dealing with model studies of spin systems, Physica B, 403, (2008) 2312-2330. (doi: 10.1016/j.physb.2007.12.011).

SJ01 A. Schweiger, G. Jeschke, Principles of Pulse Electron Paramagnetic Resonance, Oxford University Press, N.Y., 2001.

SK13 B. Sandhoefer, S. Kossmann, F. Neese. Derivation and assessment of relativistic hyperfine-coupling tensors on the basis of orbital-optimized second-order Møller-Plesset perturbation theory and the second order Douglass-Kroll-Hess transformation. J. Chem. Phys., 138 (2013) 104102. 
(doi: 10.1063/1.4792362).

SM07 D. Skrzypek, I. Madejska, J. Habdas, The characterization of cobalt(II) derivatives of selected substituted meso-tetraphenyl and tetrapyridyl porphyrins by EPR spectroscopic studies, Sol. Stat. Sc. 9 (2007) 295-302. (doi: 10.1016/j.solidstatesciences.2006.12.005)

SN92 J.M. Spaeth, J.R. Niklas, R.H, Bartram. Structural analysis of Point Defects in Solids. Spriger, Berlin, 1992

SN12 B. Sandhoefer, F. Neese. One-electron contributions to the g-tensor for second-order Douglas-Kroll-Hess theory. J. Chem. Phys., 137 (2012) 094102 (doi: 10.1063/1.4747454).

SO69 C.E. Soliverez. An effective Hamiltonian and time-independent perturbation theory. J. Phys. C: Solid State Phys. 2, (1969) 2161-2174. (doi: 10.1088/0022-3719/2/12/301).

TA77 C.P.S. Taylor, The EPR of low spin heme complexes. Relation of the $\mathrm{t}_{2 \mathrm{~g}}$ hole model and the directional properties of the g tensor and a new method for calculating the ligand field parameters, Biochim. Biophys. Acta 491 (1977) 137-149. (doi: 10.1016/0005-2795(77)90049-6).

TI64 M. Tinkham, Group Theory and Quantum Mechanics, MacGraw Hill, N.Y., 1964. Chapter 5.

TI64b M. Tinkham, Group Theory and Quantum Mechanics, MacGraw Hill, N.Y., 1964. Chapter 1.

WA99 F.A. Walker, Magnetic, spectroscopic (EPR, ESEEM, Mössbauer, MCD and NMR) studies of low-spin ferriheme centers and their corresponding heme proteins, Coord. Chem Rev. 185-186 (1999) 471-513. (doi: 10.1016/S00108545(99)00029-6).

WA04 F.A. Walker, Models of the bis-histidine-ligated electron-transferring cytochromes. Comparative geometric and electronic structure of low-spin ferro- and ferrihemes, Chem. Rev. 104 (2004) 589-615. (doi: 10.1021/cr020634j)

WB07 J.A. Weil, J.R. Bolton, Electron Paramagnetic resonance: Elementary Theory and Practical Applications. $2^{\text {nd }}$ edition, John-Wiley and Sons. Hoboken, NJ, USA, 2007. (ISBN: 978-0471-75496-1)

YH01 M. Yamaga, M. Honda, N. Kawamata, T. Fujita, K. Shimamura, T. Fukuda. Site symmetry and crystal field of $\mathrm{Ce}^{3+}$ luminescent centres in $\mathrm{KMgF}_{3}$. J. Phys.: Condens. Matter 13 (2001) 3461-3473 (doi: 10.1088/09538984/13/14/318).

ZB09 G. Zoppellaro, K.L. Bren, A.A. Ensign, E. Harbitz, R. Kaur, H.-P. Herleth, U. Ryde, L. Hederstedt, K.K. Andersson, Studies of ferric heme proteins with highly anisotropic / highly axial low spin ( $\mathrm{S}=1 / 2)$ electron paramagnetic resonance signals with bis-histidine and histidine-methionine axial iron coordination, Biopolymers 91 (2009) 1064-1082. (doi: 10.1002/bip.21267). 


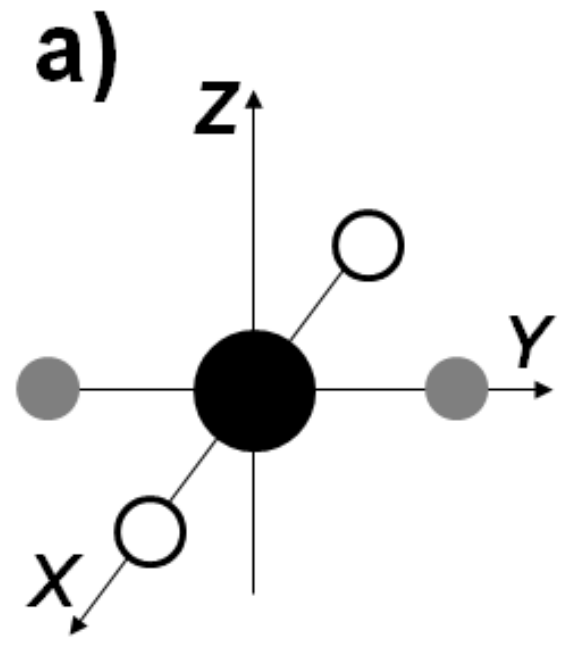

b)

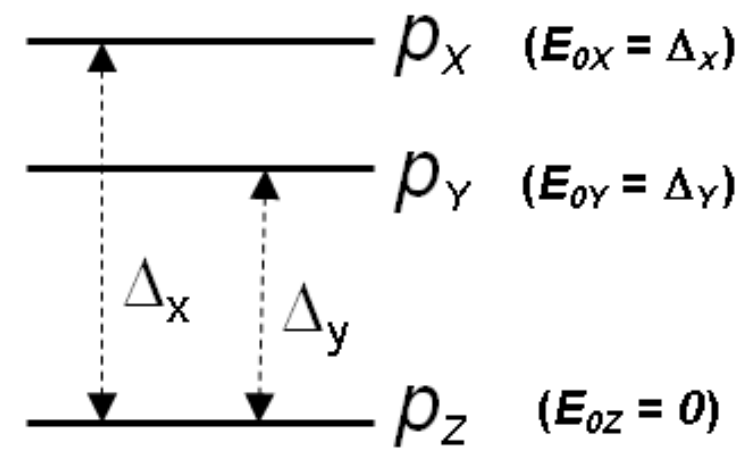

Figure 1. a) Schematic representation of the model system used in section 5. b) Orbital energy levels of the studied system showing the chosen axes labelling and the energies of the orbitals referring to the low energy one (pz), see text.

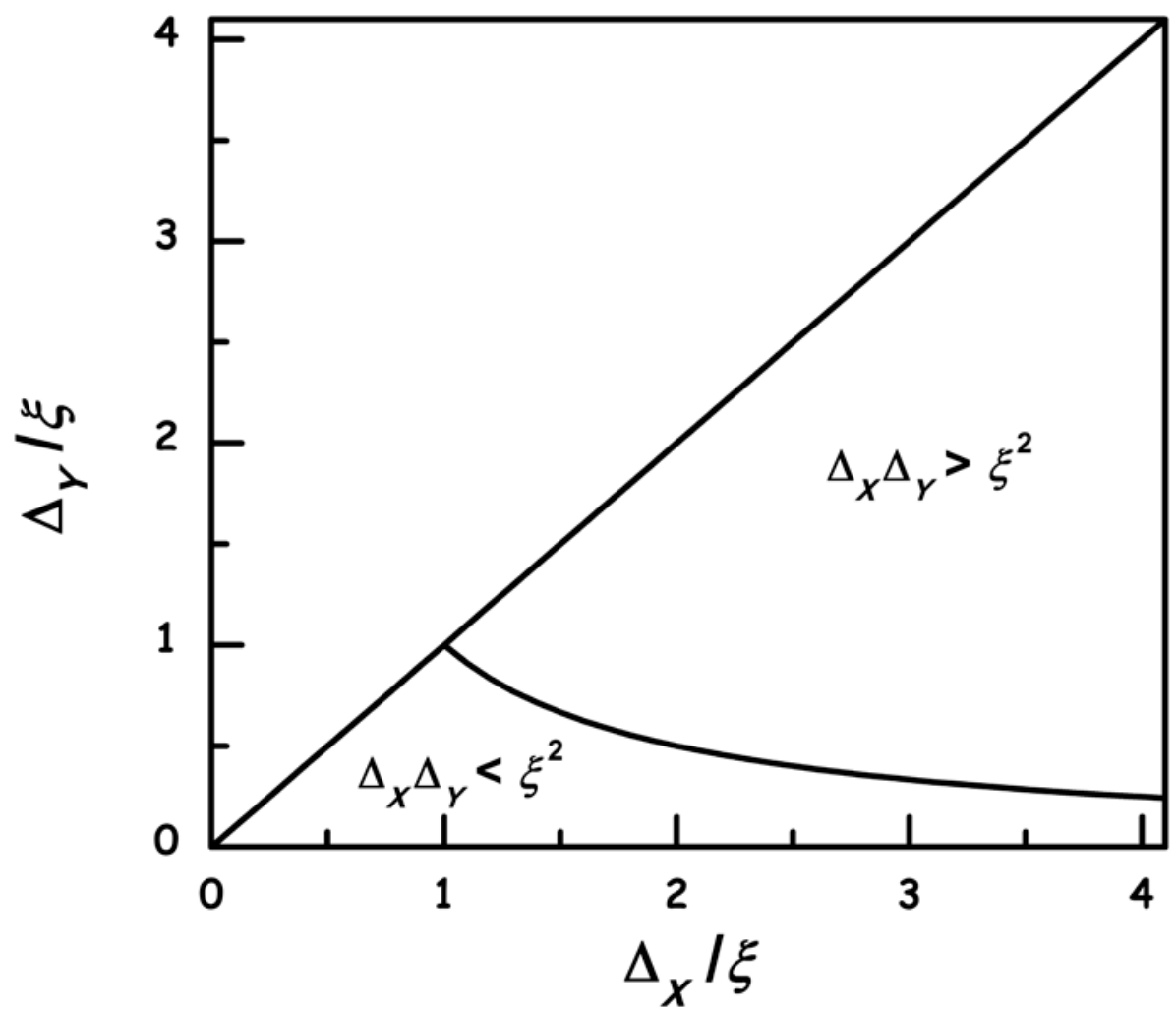

Figure 2. Regions of the $\left(\Delta_{X}, \Delta_{Y}\right)$ plane, with $\Delta_{Y}<\Delta_{X}$, in which the given description is canonical, $\Delta_{X} \Delta_{Y}>\xi$, or non-canonical, $\Delta_{X} \Delta_{Y}<\xi$. 

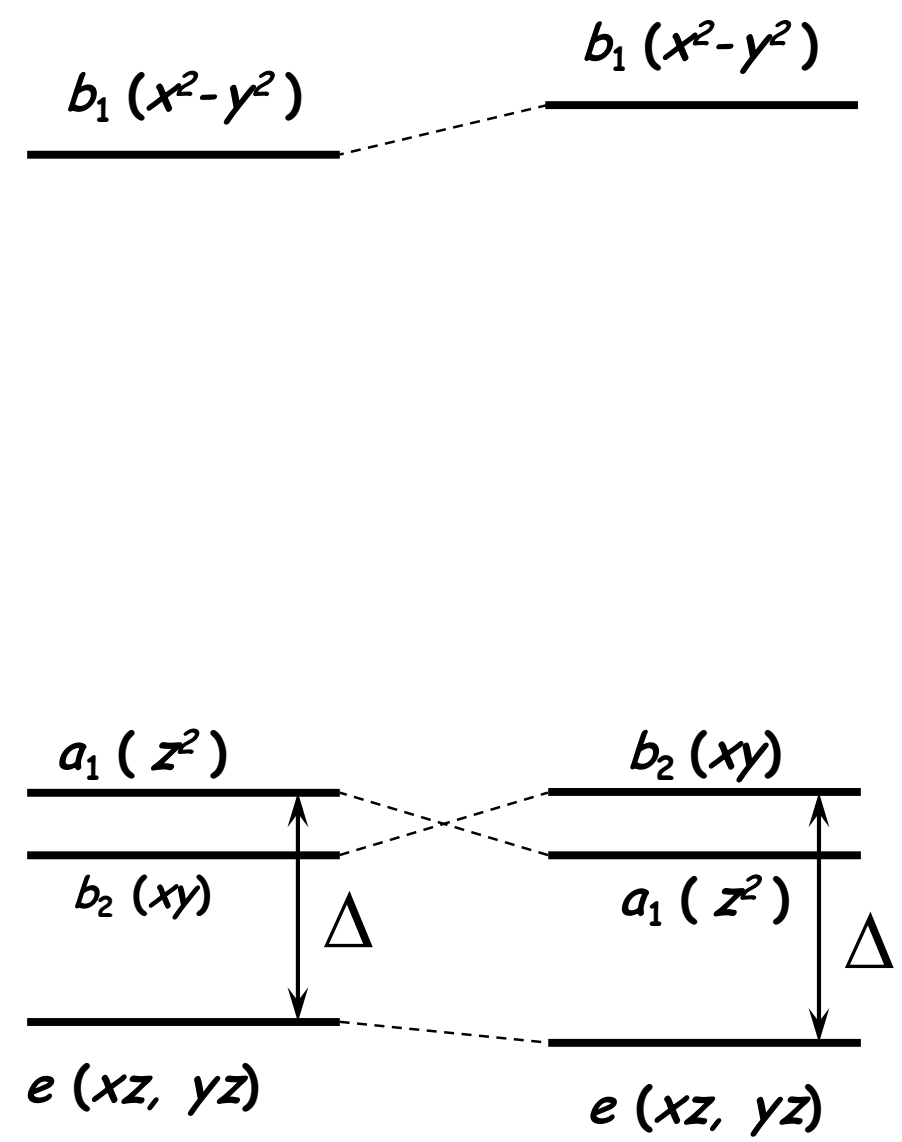

(A)

(B)

Figure 3. Single-electron energy levels in an SP-4 environment labeled according to their transformation properties under $\mathrm{D}_{4}$ symmetry operations. The two possible orderings of the $\mathbf{a}_{1}\left(z^{2}\right)$ and $b(x y)$ levels for mainly $\sigma$-donor ligands are labeled (A) and (B). The relevant energy difference $\Delta$ is indicated in each case. Adapted from reference [GA14]. 


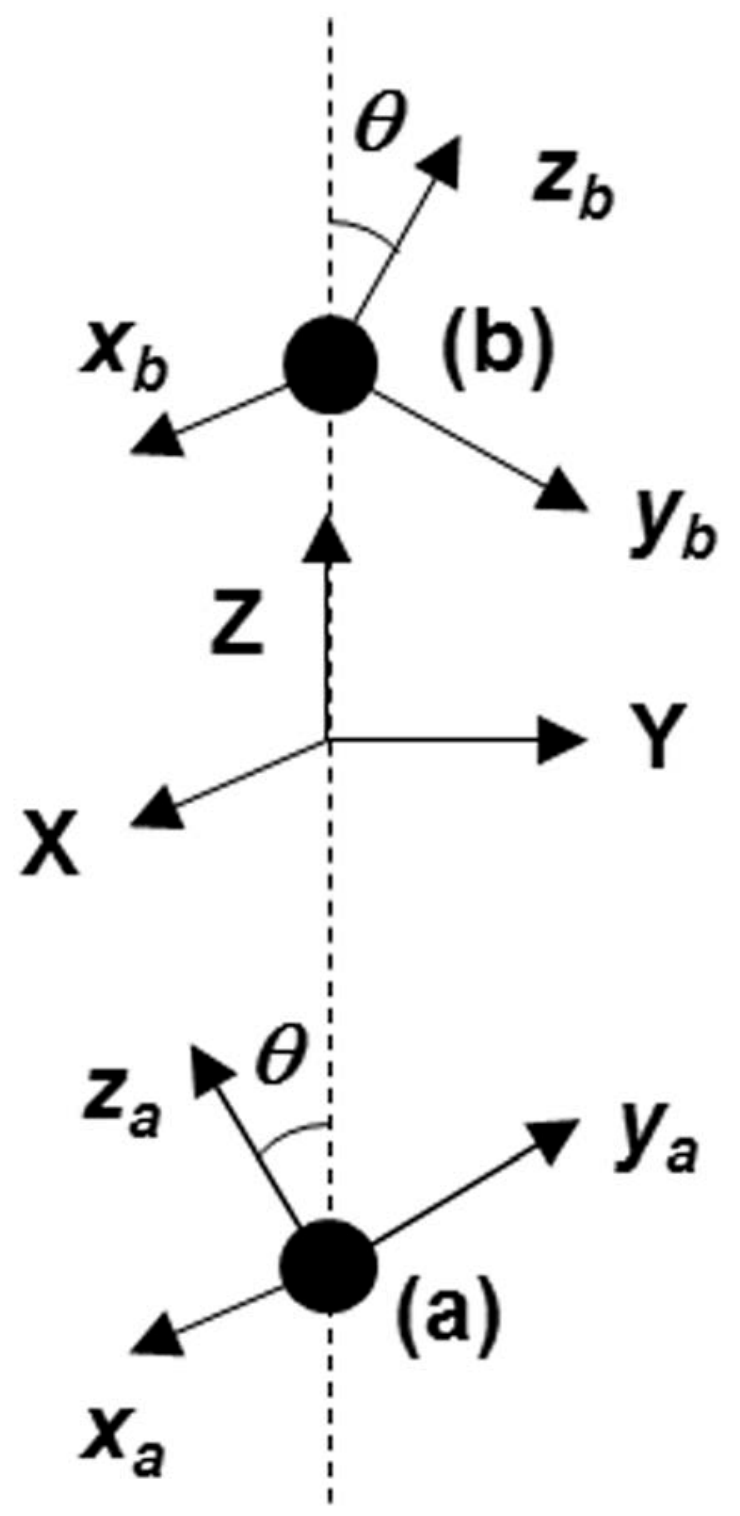

Figure 4. Sketch for the two interacting rare earth ions showing the different reference frame used. 


\title{
Supplementary information
}

for

\section{Magnetic properties of a Kramers doublet. An univocal bridge between experimental results and theoretical predictions.}

\begin{abstract}
P. J. Alonso and J. I. Martínez
Instituto de Ciencia de Materiales de Aragón (Universidad de Zaragoza-Consejo Superior de Investigaciones Científicas) Facultad de Ciencias, Universidad de Zaragoza, C/ Pedro Cerbuna 12, 50009 Zaragoza (Spain)
\end{abstract}

The Supplementary information contents i) a detailed derivation of the effective spin formalism for describing a Kramers doublet. ii) detailed analysis of the example given in section 5 iii) derivation of the $g$-tensor of a LS-Fe(III) system and its relation with the Taylor's equations. 


\section{S.1. Fictitious spin formalism for describing a Kramers doublet.}

A paradigm of a Kramers doublet is an $S=1 / 2$ system. The corresponding state space will be denoted by $\mathbf{S}_{\mathbf{1} / 2}$. Given any reference frame $\varsigma \equiv(x, y, z)$ it is possible to take a KCB of $\mathbf{S}_{1 / 2},\{|\alpha\rangle,|\beta\rangle\}$, of eigenstates of $S_{z}$ and, with a suitable choice of phase, the matrix expressions of the spin components $S_{r}(j=x, y, z)$ in this basis are multiples (by a $1 / 2$ factor) of the Pauli matrices. On the other hand, given a KD we can pick any $\mathrm{KCB}\{\Psi, T \Psi\}$ to describe it. Then, an isometry, $\lambda$, between the $\mathrm{KD}$ and $\mathbf{S}_{\mathbf{1} / 2}$ can be defined by $\lambda(\Psi)=|\alpha\rangle$ and $\lambda(T \Psi)=|\beta\rangle$. Associated to this isometry an isomorphism $\Lambda, \Lambda(A)=\lambda \cdot A \lambda^{-1}$, appears between the algebra of endomorphisms of $\mathrm{KD}$ and the algebra of endomorphisms of $\mathbf{S}_{\mathbf{1} / \mathbf{2}}$, which preserves the trace of any operator. In short, $\Lambda$ is an isometry between the corresponding Liouville spaces that transforms an observable into an observable and a unitary operator into a unitary operator. $\Lambda$ also transforms the time reversal operator in the $\mathrm{KD}$ into the time reversal operator in $\mathbf{S}_{1 / 2}$. The notation $A^{S}$ for $\Lambda(A)$ will be used in the following. Operator $A$ acting on the physical states in the $\mathrm{KD}$ is transformed into $A^{S}=\Lambda(A)$ acting in the $\mathbf{S}_{\mathbf{1} / \mathbf{2}}$ space. On the other hand, taking into account the freedom in the choice of the KCB of KD (and in the choice of the reference frame in the $\mathbf{S}_{\mathbf{1} / \mathbf{2}}$ description) there are many ways to define $\lambda$ corresponding to different representations [S1-S2].

In particular, fixing a reference frame, $\Sigma_{0}\left(X_{0}, Y_{0}, Z_{0}\right)$, and a $\operatorname{KCB}\left\{\Psi_{0}, \mathrm{~T} \Psi_{0}\right\}$ that give a canonical description of the $\mathrm{KD}$ (see paragraph 3) and referring the $\{|\alpha\rangle$, $|\beta\rangle\} \mathrm{KCB}$ of $\mathbf{S}_{1 / 2}$ to the same reference frame, $\Sigma_{0}$, it follows that:

$$
n_{X_{0}}^{S}=\sqrt{2} S_{X_{0}} \quad n_{Y_{0}}^{S}=\sqrt{2} S_{Y_{0}} \quad n_{Y_{0}}^{S}=\sqrt{2} S_{Z_{0}}
$$

where $n_{p}\left(p=X_{0}, Y_{0}, Z_{0}\right)$ are the components, in the $\Sigma_{0}$ reference frame, of the vector operator $\vec{n}$ defined in section 3. Since a basis of the Liouville space of the KD is given by the identity and the components of the vector operator $\vec{n}$ in any reference space, (S.1) provides a practical rule to build the transformed operator, $A^{S}$, of any operator $A$. The rule is given by the following substitution law

$$
\vec{n} \leftarrow \rightarrow \vec{n}^{S}=\sqrt{2} \vec{S}
$$

In particular, from (26), it follows that: 


$$
\Lambda\left(H_{Z e}\right)=H_{Z e}^{S}=\mu_{B} \vec{B} \cdot \tilde{g} \cdot \vec{S}
$$

and the equivalent operator of the spin density matrix, $\rho$, of the physical $\mathrm{KD}$, which act on $\mathrm{S}_{1 / 2}$, results:

$$
\rho^{S}=\Lambda(\rho)=\lambda \cdot\left(\frac{e^{-H_{Z e} / k_{B} \theta}}{\operatorname{tr}\left(e^{-H_{Z e} k_{B} \theta}\right)}\right) \cdot \lambda^{-1}=\frac{\lambda \cdot e^{-H_{Z e} k_{B} \theta} \cdot \lambda^{-1}}{\operatorname{tr}\left(\cdot \lambda e^{-H_{Z e} k_{B} \theta} \cdot \lambda^{-1}\right)}=\frac{e^{-\Lambda\left(H_{Z e}\right) k_{B} \theta}}{\operatorname{tr}\left(e^{-\Lambda\left(H_{Z e}\right) k_{B} \theta}\right)}=\frac{e^{-H_{Z e}^{S} / k_{B} \theta}}{\operatorname{tr}\left(e^{-H_{Z e}^{S} / k_{B} \theta}\right)}
$$

where $\theta$ indicates the temperature. It is important to realize that $\rho^{S}$ coincides with the spin density operator in $\mathbf{S}_{\mathbf{1} / \mathbf{2}}$ associated to the Hamiltonian (S.3).

Let $O$ be an observable in the KD. Its expectation value is given by $\langle O\rangle=\operatorname{tr}(\rho O)$. Since $\operatorname{tr}(\rho O)=\operatorname{tr}\left(\rho \lambda^{-1} \lambda O \lambda^{-1} \lambda\right)=\operatorname{tr}\left(\lambda \rho \lambda^{-1} \lambda O \lambda^{-1}\right)=\operatorname{tr}\left(\rho^{S} O^{S}\right)$, this expectation value coincides with the expectation value of the observable $O^{S}=\Lambda(O)$ in $\mathbf{S}_{\mathbf{1} 2 \mathbf{2}}$ under the Hamiltonian (S.3). As a consequence any observation on the physical KD in an arbitrary reference system should coincide with that of the $\mathbf{S}_{\mathbf{1} / 2}$ system under the Hamiltonian (S.3) if the relationship of the observables given above is taken into account.

\section{S.2. Detailed analysis of the example given in section 5}

In section 5 we introduce a particular example that show why information on the ligand field (LF) cannot be univocally derived from the measured $g$-tensor in a general situation. Here we present a detailed analysis of this model. We refer in the following to the initial reference frame, $\Sigma \equiv(\mathrm{X}, \mathrm{Y}, \mathrm{Z})$, and to the LF splitting defined in figure 1 . In the following we will use the convention of taking the energy of the ground orbital, $p_{z}$ equal to zero (see figure 1).

In absence of spin-orbit (SO) interaction the ground state is a KD that can be described by the $\operatorname{KCB}\left\{p_{z} \alpha, p_{z} \beta\right\}$ where $\alpha$ and $\beta$ denote the spin states corresponding to $m_{s}=+1 / 2$ and $m_{s}=-1 / 2$, respectively, referred to the $Z$ axis. This KD behaves as an $S=1 / 2$ entity with and isotropic $g$-factor ( $g=g_{e}$ where $g_{e}$ is the free electron $g$-factor).

Let us start considering a situation where the spin-orbit coupling constant (SOCC), $\xi$, is small as compare with the LF energy differences, $\Delta_{X}$ and $\Delta_{Y}$. So, the SO interaction, $H_{S O}=\xi \vec{l} \cdot \vec{s}$, is cinsidered up to a first order. The energies of the levels do not change since the orbital angular momentum is quenched in each of the $p_{r}(r=X, Y, Z)$ orbitals. However, 
the wave function is modified by the mixing with the excited states induced by $H_{s o}$. The perturbed wave-functions that span the ground KD are given, up to a first order in $\xi$, by:

$$
\begin{aligned}
& \Phi^{+}=\left|p_{Z}, \alpha\right\rangle+\frac{i}{2} \frac{\xi}{\Delta_{y}}\left|p_{Y}, \beta\right\rangle+\frac{1}{2} \frac{\xi}{\Delta_{x}} \mid p_{X}, \beta \\
& \Phi^{-}=\left|p_{Z}, \beta\right\rangle+\frac{i}{2} \frac{\xi}{\Delta_{y}}\left|p_{Y}, \alpha\right\rangle-\frac{1}{2} \frac{\xi}{\Delta_{x}} \mid p_{X}, \alpha
\end{aligned}
$$

As $\Phi^{-}=T \Phi^{+}$, (S.5) provides a KCB of the ground state KD. Using this KCB and the reference frame, $\Sigma \equiv(X, Y, Z)$, depicted in figure 1 , the $G$ matrix, see equation (5), can be built. Keeping linear terms in $\xi / \Delta_{X}$ and $\xi / \Delta_{Y}$ :

$$
G_{\Sigma, \Phi}=\left(\begin{array}{ccc}
2-\frac{\xi}{\Delta_{X}} & 0 & 0 \\
0 & 2-\frac{\xi}{\Delta_{Y}} & 0 \\
0 & 0 & 2
\end{array}\right)
$$

Then, $G_{\Sigma, \Phi}$ is diagonal and all its diagonal elements have the same sign because it is assumed that $H_{S O}$ is a perturbation on the ligand field states $\left(\xi / \Delta_{X}, \xi / \Delta_{Y}<<1\right)$. So, the KCB given by (S.5) and the reference frame $\Sigma \equiv(\mathrm{X}, \mathrm{Y}, \mathrm{Z}$ ) (see Figure 1) provide a canonical description of the ground KD. Consequently, see section 3, a $g$-tensor can be defined for describing the EPR results. Its principal axes coincide with $(X, Y, Z)$ axes and their principal values are:

$$
g_{X}=2-\frac{\xi}{\Delta_{X}} \quad g_{Y}=2-\frac{\xi}{\Delta_{Y}} \quad g_{z}=2
$$

So, an EPR experiment provides information about the ligand field splitting if the spin-orbit interaction is low enough as compared to $\Delta_{X}$ and $\Delta_{Y}$.

The second order contribution of $H_{\text {so }}$ to the energy of the ground doublet is given by:

$$
E_{o}^{(2)}=-\frac{1}{4} \xi^{2}\left(\frac{1}{\Delta_{X}}+\frac{1}{\Delta_{Z}}\right)
$$

Note that it is negative whereas the energies of the excited doublets are positive in the strong $\operatorname{LF} \operatorname{limit}\left(\Delta_{X}, \Delta_{Y}>>\xi\right)$. 
The opposite occurs when the SO interaction is dominant. In particular, when $\Delta_{X}=\Delta_{Y}$ $=0$, the ground doublet energy is $E_{0}=-\xi$ and there are two degenerate Kramers doublets with energy $E_{1}=\xi / 2$. Note that whereas $E_{0}$ is negative $E_{1}$ is positive. A KCB of the ground state is given by:

$$
\begin{aligned}
& \left|\phi_{0}^{+}\right\rangle=\frac{1}{\sqrt{3}}\left\{\left|p_{Z} \alpha\right\rangle+i\left|p_{Y} \beta+\right| p_{X} \beta\right\} \\
& \left|\phi_{0}^{-}\right\rangle=\frac{1}{\sqrt{3}}\left\{\left|p_{Z} \beta\right\rangle+i\left|p_{Y} \alpha\right\rangle-\mid p_{X} \alpha\right\}
\end{aligned}
$$

For a low enough ligand field contribution $\left(\Delta_{X}, \Delta_{Y}<<\xi\right)$ the energy of the ground doublet, up to a first order, is

$$
E_{0}^{(1)}=-\xi+\frac{1}{3}\left(\Delta_{X}+\Delta_{Y}\right)
$$

Note that it is negative, $E_{0}^{(1)}<0$.

The question now is to analyse what happens when both, $H_{S O}$ and the ligand field, $H_{L F}$, contributions are comparable. In such a situation, it will be necessary to simultaneously consider $H_{L F}$ and $H_{S O}$. As long as we are dealing with a Kramers system a KCB of the ground state can be given by:

$$
\begin{gathered}
\Psi^{+}=u\left|p_{Z}, \alpha+i v\right| p_{Y}, \beta+w\left|p_{X}, \beta\right\rangle \\
\left.\Psi^{-}=u\left|p_{Z}, \beta+i v\right| p_{Y}, \alpha\right\rangle+w\left|-p_{X}, \alpha\right\rangle
\end{gathered}
$$

where $(u, v, w)$ are non-trivial real solutions of:

$$
H\left(\begin{array}{l}
u \\
v \\
w
\end{array}\right)=\xi\left(\begin{array}{ccc}
0 & -1 / 2 & -1 / 2 \\
-1 / 2 & \Delta_{Y} / \xi & -1 / 2 \\
-1 / 2 & -1 / 2 & \Delta_{X} / \xi
\end{array}\right)\left(\begin{array}{l}
u \\
v \\
w
\end{array}\right)=E\left(\begin{array}{l}
u \\
v \\
w
\end{array}\right)
$$

corresponding to the lowest $E / \xi$ value. Since $H$ is a symmetric real matrix, the $(u, v, w)$ coefficients were chosen to be real with $u^{2}+v^{2}+w^{2}=1$. It worth of noting that $E=0$ is not a solution of (S.12) regardless of what the values of $\Delta_{X} / \xi$ and $\Delta_{Y} / \xi$ are, since $\operatorname{det}(H)=$ $-(1 / 4) \xi^{2}\left(\xi+\Delta_{X}+\Delta_{Y}\right) \neq 0$ (remember that the origin of energy has been conventionally taken in the $p_{z}$ orbital, see figure 1). Consequently, that lowest $E / \xi$ value is negative for any value of the $\Delta_{X} / \xi$ and $\Delta_{Y} / \xi$ ratios since the eigenvalues of $H$ are continuous functions of $\left(\xi, \Delta_{X}, \Delta_{Y}\right)$.

From (S.12) it follows that: 


$$
\frac{E}{\xi}=-\frac{1}{2} \frac{v+w}{u} \quad \frac{\Delta_{Y}}{\xi}=\frac{1}{2}(u+v+w)\left(\frac{1}{v}-\frac{1}{u}\right) \quad \frac{\Delta_{X}}{\xi}=\frac{1}{2}(u+v+w)\left(\frac{1}{w}-\frac{1}{u}\right)
$$

Taking $u>0$, since $E / \xi<0$, it follows that $(v+w)>0$, and also $(u+v+w)>0$. Besides, $1 / w$ $\geq 1 / v \geq 1 / u$, since $\Delta_{X} / \xi \geq \Delta_{Y} / \xi \geq 0$ with the axes labelling shown in figure 1 . So:

$$
u>v \geq w \geq 0
$$

Using the KCB given in (S.11) and the reference frame $\Sigma \equiv(\mathrm{X}, \mathrm{Y}, \mathrm{Z})$ depicted in figure 1.a the matrix $G_{\Sigma, \Psi}$ is again diagonal, with:

$$
\begin{aligned}
& G_{X X}=2\left(u^{2}+v^{2}-w^{2}-2 u v\right)=2\left\{(u-v)^{2}-w^{2}\right\}=2(u-v-w)(u-v+w) \\
& G_{Y Y}=2\left(u^{2}-v^{2}+w^{2}-2 u w\right)=2\left\{(u-w)^{2}-v^{2}\right\}=2(u-v-w)(u+v-w) \\
& G_{Z Z}=2\left(u^{2}-v^{2}-w^{2}+2 v w\right)=2\left\{u^{2}-(v-w)^{2}\right\}=2(u-v+w)(u+v-w)
\end{aligned}
$$

where we have taken $g_{e}=2$. These expressions correspond to equation (33) in section 5 .

\section{S.3. The $g$-tensor of a LS-Fe(III) systems. Relation with the Taylor's equations}

As indicated in section 6.1 the description, of the magnetic properties of LS-Fe(III) systems has been the goal of many works since the pioneer paper by Bleaney and O’Brian [S3]. We have also commented that the formalism developed in section 3 is useful to this issue and the main derived conclusions were also given there. Here we will show technical details about how to define a $g$-tensor for this system by applying the developed formalism. As some steps are given in reference [S3] and some others are similar to those addressed in the previous section (Supplementary Information, S.2) many details will be omitting for the sake of brevity.

It should be remembered that the strong ligand field (LF) scheme with a dominant octahedral contribution is commonly assumed for describing the electronic properties of these LS-Fe(III) systems; the five $d$ electrons are accommodated in the six available orbitals of the $t_{2 g}$ triplet and excitations to the empty $e_{g}$ orbitals are neglected [S4]. This octahedral LF contribution implicitly defines a reference frame, $\varsigma \equiv(x, y, z)$, that will be denoted as molecular reference frame. Hence, the lowest energy $t_{2 g}$ triplet is spanned by the $\left\{d_{y z}, d_{z x}, d_{x y}\right\}$ standard orbitals. 
When the low symmetry LF contribution are considered up to a first order the $t_{2 g}$ triplet is split and the relevant energy differences are commonly given as a function of two parameters, $\Delta$ and $V$ (see figure S.1. and reference [S4] for details). Besides, the LF orbital wave functions $\left\{\phi_{X}, \phi_{Y}, \phi_{Z}\right\}$ are expressed as a linear combination of the $\left\{d_{y z}, d_{z x}, d_{x y}\right\}$ standard orbitals by defining an direct orthogonal matrix $\Omega \in \mathbf{S O}(3)$. In this way a reference frame $\Sigma \equiv(X, Y, Z)$ is introduced by:

$$
\left(\begin{array}{l}
X \\
Y \\
Z
\end{array}\right)=\Omega \cdot\left(\begin{array}{l}
x \\
y \\
z
\end{array}\right)
$$

which is commonly named as magnetic reference frame, because it coincide with the $g$-tensor principal axes as it will be seen below.

Using the hole formalism [S4] the problem may be reduced to a single-particle one. In figure S.1.b the energy levels for the hole are shown and, for convenience, the relevant hole energy differences, $\Delta_{Y}$ and $\Delta_{Z}$, are also indicated. These latter parameters are given as a function of the former ones, $\Delta$ and $V$, by:

$$
\begin{array}{cc}
\Delta_{Z}=\Delta+\frac{1}{2} V & \Delta=\Delta_{Z}-\frac{1}{2} \Delta_{Y} \\
\Delta_{Y}=V & V=\Delta_{Y}
\end{array}
$$

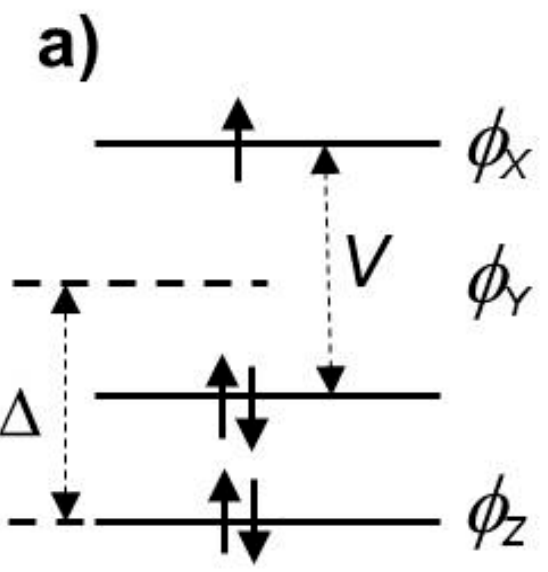

b)

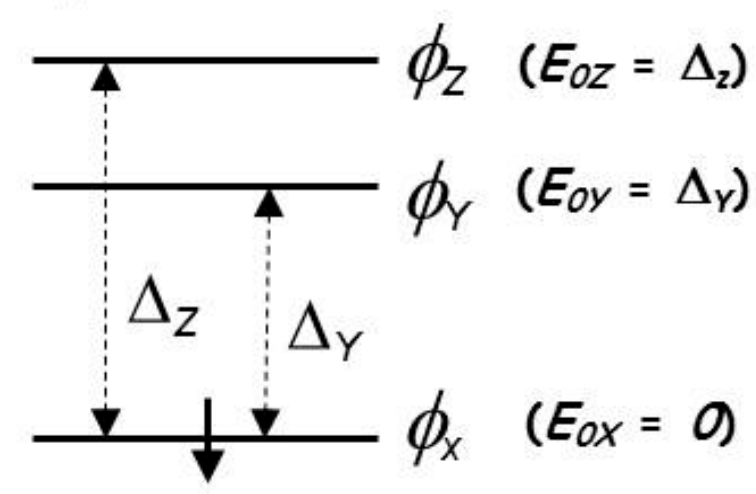

Figure S.1. a) Low lying energy levels of a LS- $d^{5}$ entity showing the electron filling. b) 
Energy levels for the equivalent hole. The relevant energy differences $\left(\Delta_{y}, \Delta_{z}\right)$ and the equivalent conventional $\operatorname{LF}(\Delta, V)$ parameters are also shown.

Note that, as in section S.2, we adopt the labelling of the $(X, Y, Z)$ axes imposing the additional condition $\Delta_{Z} \geq \Delta_{Y} \geq 0$ or, equivalently, $\Delta \geq V / 2 \geq 0$. Then, the hole ground orbital is $\phi_{X}$ and, for convenience in the calculation, the hole energy will be referred to it $\left(E_{0 X}=0\right.$, see figure S.1). This labelling may not correspond to the proper axes convention widely adopted in the description of heminic systems (see, for instance [S4 -S6]), which is imposed by $0 \leq V$ $\leq 2|\Delta| / 3$.

When the SO interaction is taking into account a KSB of the ground $\mathrm{KD}$ is given by:

$$
\begin{gathered}
\left.|\Phi\rangle=a\left|\phi_{X} \alpha-i b\right| \phi_{Y} \alpha\right\rangle-c\left|\phi_{Z} \beta\right\rangle \\
|T \Phi\rangle=a\left|\phi_{X} \beta+i b\right| \phi_{Y} \beta+c\left|\phi_{Z} \alpha\right\rangle
\end{gathered}
$$

where the spin function $\{\alpha, \beta\}$ are referred to the formerly introduced reference frame $\Sigma \equiv(X, Y, Z)$ and $(a, b, c)$ are the non-trivial real solutions of:

$$
H\left(\begin{array}{l}
a \\
b \\
c
\end{array}\right)=\left(\begin{array}{ccc}
0 & -\frac{1}{2} \zeta & -\frac{1}{2} \zeta \\
-\frac{1}{2} \zeta & \Delta_{Y} & -\frac{1}{2} \zeta \\
-\frac{1}{2} \zeta & -\frac{1}{2} \zeta & \Delta_{Z}
\end{array}\right)\left(\begin{array}{l}
a \\
b \\
c
\end{array}\right)=E\left(\begin{array}{l}
a \\
b \\
c
\end{array}\right)
$$

corresponding to the lowest eigenvalue of $H, E_{0}$, In (S.19) $\zeta$ is the single electron SOCC.

Following the same arguments as in section S.2 it is proved that $E_{0} \leq 0$ (with the energy convention given in figure S.1). Moreover, once the coefficients $(a, b, c)$ were known, the relevant energies relative to the SOCC, $\Delta_{Y} / \zeta, \Delta_{Z} / \zeta$ and $E_{0} / \zeta$ result to be:

$$
\frac{E_{0}}{\zeta}=-\frac{1}{2} \frac{b+c}{a} \quad \frac{\Delta_{Y}}{\zeta}=\frac{1}{2}(a+b+c) \frac{a-b}{a b} \quad \frac{\Delta_{Z}}{\zeta}=\frac{1}{2}(a+b+c) \frac{a-c}{a c}
$$

and taking $a>0$ it follows

$$
a>b \geq c \geq 0
$$

Note that $b=c$ if and only if $\Delta_{Z}=\Delta_{Y}$, which corresponds to an axial situation. In an isotropic environment $\Delta_{Z}=\Delta_{Y}=0$ and $a=b=c=1 / \sqrt{ } 3$, see above. 
When the KCB given by (S.18) and the $\Sigma \equiv(X, Y, Z)$ reference frame are used for calculating the $G$-matrix, it results;

$$
G_{\Sigma, \Phi}=-2 g_{e}\left(\begin{array}{lll}
a^{2}-b^{2}-c^{2} & & \\
& a^{2}-b^{2}+c^{2} & \\
& & a^{2}+b^{2}-c^{2}
\end{array}\right)-2\left(\begin{array}{lll}
-4 b c & & \\
& 4 a c & \\
& & 4 a b
\end{array}\right)
$$

So, the $G_{\Sigma, \Phi}$-matrix is diagonal and consequently the $\Sigma \equiv(X, Y, Z)$ axes correspond to the principal axes of the $g$-tensor (see section 3). If $g_{e}=2$ is taken the principal values of the $G_{\Sigma, \Phi}$-matrix accomplish

$$
\begin{aligned}
G_{X X} & =-2(a+b+c)(a-b-c) \\
G_{Y Y} & ==-2(a+b+c)(a-b+c) \\
G_{Z Z} & ==-2(a+b+c)(a+b-c)
\end{aligned}
$$

From (S.23) it follows that

$$
\frac{a}{G_{Z Z}+G_{Y Y}}=\frac{b}{G_{Z Z}-G_{X X}}=\frac{c}{G_{Y Y}-G_{X X}}
$$

This, together the normalization condition, $a^{2}+b^{2}+c^{2}=1$, and the convention $a>0$, would yield a univocal determination of the coefficients $(a, b, c)$ if the $\left(G_{X X}, G_{Y Y}, G_{Z Z}\right)$ values were known. Then, the energy splittings relative to the spin-orbit coupling constant are obtained after using (S.20). On the other hand, since $\operatorname{det}(G)=G_{X X} G_{Y Y} G_{Z Z}$, its sign, $s$, is determined by the sign of $(a-b-c)$; $s=-\operatorname{sign}(a-b-c)$. It will now be discussed what information about the $G$-values can be obtained from an EPR experiment.

Taking into account (S.21) we have $G_{Y Y} \leq 0$ and $G_{Z Z} \leq 0$ with $\left|G_{Y Y}\right| \leq\left|G_{Z Z}\right|$ in all cases whereas the sign of $G_{X X}$ is determined by the $(a-b-c)$ value. Consequently, the previous description is canonical if $(a-b-c) \geq 0$ and non-canonical when $(a-b-c)<0$. The condition $(a-b-c)=0$ will define the regions of the $\left(\Delta_{Z} / \zeta, \Delta_{Y} / \zeta\right)$ plane where the previous description is canonical or not. From (S.20), the condition $(a-b-c)=0$ is equivalent to $\Delta_{Z} \Delta_{Y}$ $=\zeta^{2}$; the previous description is canonical if $\Delta_{Z} \Delta_{Y}>\zeta^{2}$ whereas it is non-canonical if $\Delta_{Z} \Delta_{Y}<$ $\zeta^{2}$. This result is similar to that found in the case of the example discussed in section 5 by exchange $\Delta_{z}$ by $\Delta_{X}$ (see Figure 2). In terms of the ligand field parameters, $\Delta$ and $V$, conventionally used (in the heminic system context) for describing the ligand field effects, see 
(S.17), the previous description is canonical if $(\Delta+V / 2) V>\zeta^{2}$ whereas it is non-canonical in the opposite case. This is illustrated in figure S.2.

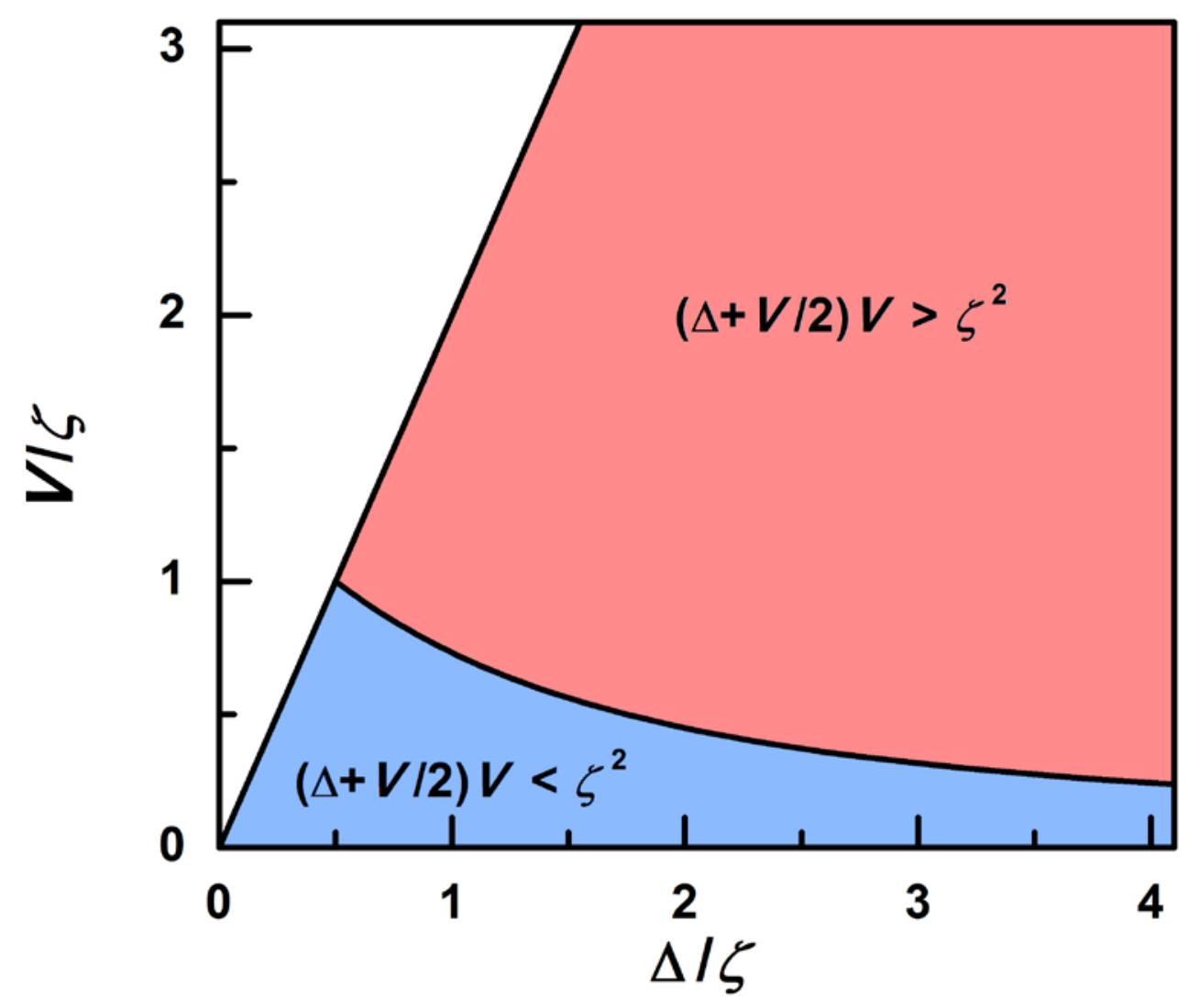

Figure S.2. Regions of the $(V, \Delta)$ plane, with $V<2 \Delta$, in which the given description is canonical (red shaded) or it is non-canonical (blue shaded).

Assuming that the above description using the KCB given by (S.18) together the $\Sigma \equiv$ $(X, Y, Z)$ reference frame is canonical, $(a-b-c) \geq 0$, which implies $G_{X X} \leq 0$; also $\left|G_{X X}\right| \leq\left|G_{Y Y}\right|$ $\leq\left|G_{z z}\right|$ and in this case the principal values of the $g$-tensor are all negative fulfilling

$$
G_{X X}=-\left|g_{X}\right| \quad G_{Y Y}=-\left|g_{Y}\right| \quad G_{Z Z}=-\left|g_{Z}\right|
$$

Then, from (S.24), it is explicitly obtained that 


$$
\begin{aligned}
& a=\frac{\left|g_{Y}\right|+\left|g_{Z}\right|}{\sqrt{\left.\left|g_{Y}\right|+\left|g_{Z}\right|\right)^{2}+\left(\left|g_{Z}\right|-\left|g_{X}\right|\right)^{2}+\left(\left|g_{Y}\right|-\left|g_{X}\right|\right)^{2}}} \\
& b=\frac{\left|g_{Z}\right|-\left|g_{X}\right|}{\sqrt{\left(g_{Y}|+| g_{Z} \mid\right)^{2}+\left(\left|g_{Z}\right|-\mid g_{X}\right)^{2}+\left(\left|g_{Y}\right|-\mid g_{X}\right)^{2}}} \\
& c=\frac{\left|g_{Y}\right|-\left|g_{X}\right|}{\sqrt{\left(g_{Y}|+| g_{Z} \mid\right)^{2}+\left(g_{Z}|-| g_{X} \mid\right)^{2}+\left(g_{Y}|-| g_{X}\right)^{2}}}
\end{aligned}
$$

In the opposite case, $(a-b-c)<0$, the previous description is non-canonical since $G_{X X}>0$ while $G_{Y Y} \leq 0$ and $G_{Z Z} \leq 0$. Consequently $\operatorname{det}(G)>0$ and all the principal values of the $g$-tensor are positive. A canonical description is achieved by keeping the $\Sigma \equiv(X, Y, Z)$ reference frame and by choosing a new KCB defined by $\{|+>|-,>\}$ with $|+>=i T| \Phi>(\mid->=$ $i \mid \Phi>$ ) where $\mid \Phi>$ is given by (S.18). Then:

$$
\begin{aligned}
G_{X X} & =-2(a+b+c)(a-b-c) \\
G_{Y Y} & =+2(a+b+c)(a-b+c) \\
G_{Z Z} & =+2(a+b+c)(a+b-c)
\end{aligned}
$$

with $0<G_{X X} \leq G_{Y Y} \leq G_{Z Z}$; in this case

$$
G_{X X}=\left|g_{X}\right| \quad G_{Y Y}=\left|g_{Y}\right| \quad G_{Z Z}=\left|g_{Z}\right|
$$

Consequently if $\Delta_{Z} \Delta_{Y}<\zeta^{2}$ the coefficients $(a, b, c)$ that describe the ground KD are given as a function of the principal value of the $g$-tensor as:

$$
\begin{aligned}
& a=\frac{\left|g_{Y}\right|+\left|g_{Z}\right|}{\sqrt{\left.\left|g_{Y}\right|+\left|g_{Z}\right|\right)^{2}+\left(\left|g_{Z}\right|+\left|g_{X}\right|\right)^{2}+\left(\left|g_{Y}\right|+\left|g_{X}\right|\right)^{2}}} \\
& b=\frac{\left|g_{Z}\right|+\left|g_{X}\right|}{\sqrt{\left(g_{Y}|+| g_{Z} \mid\right)^{2}+\left(g_{Z}|+| g_{X} \mid\right)^{2}+\left(\left|g_{Y}\right|+\left|g_{X}\right|\right)^{2}}} \\
& c=\frac{\left|g_{Y}\right|+\left|g_{X}\right|}{\sqrt{\left(g_{Y}|+| g_{Z} \mid\right)^{2}+\left(g_{Z}|+| g_{X} \mid\right)^{2}+\left(g_{Y}|+| g_{X}\right)^{2}}}
\end{aligned}
$$

It is important to recall that a conventional EPR experiment only provides the absolute values $\left|g_{x}\right|,\left|g_{x}\right|,\left|g_{z}\right|$. Therefore, such an experiment cannot discriminate between the two above described situations and it provides, in principle, two solutions for the description of the 
ground $\mathrm{KD}$, corresponding to expressions (S.26), that coincide with the equations derived by Taylor [S5] and to expressions (S.29).

This ambiguity is similar to that studied in the example discussed in section 5 . However, when the sign of the product $g_{x} g_{x} g_{z}$, that coincide with the sign of $\operatorname{det}(G)$, is established from an adequate experiment (i.e. by taking the EPR spectrum using circular polarized microwave and measuring the difference of the signals corresponding to the two opposite polarizations [S7-S9]) all ambiguities are overcome and univocal information about the ligand field splittings relative to the spin-orbit coupling constant can be obtained. The finding $g_{x} g_{x} g_{z}>0$ implies $(\Delta+V / 2) V>\zeta^{2}$ whereas $g_{x} g_{x} g_{z}<0$ entails $(\Delta+V / 2) V>\zeta^{2}$.

\section{REFERENCES}

[S1] A. Messiah, Mécanique Quantique, Dunod, Paris, 1964. Part I, page 272.

[S2] M.J. Caola, The spin Hamiltonian, J. Phys. A: Gen. Phys. 5 (1972) 1649-1657. (doi: 10.1088/0305-4470/5/12/003).

[S3] B. Bleaney, M.C.M. O’Brian, paramagnetic resonance of some complex cyanides of the iorn group. II theory, Proc. Phys. Soc. B69, (1956) 1216-1230. (doi: 10.1088/0370-1301/69/12/305).

[S4] P.J. Alonso, J.I. Martínez, I. García-Rubio, The study of the ground state Kramers doublet of low heminic system revisited. A comprehensive description of the EPR and Mössbauer spectra, Coord. Chem. Rev. 251 (2007) 12-24. (doi: 10.1016/j.ccr.2006.05.007).

[S5] C.P.S. Taylor, The EPR of low spin heme complexes. Relation of the $t_{2 g}$ hole model and the directional properties of the g tensor and a new method for calculating the ligand field parameters, Biochim. Biophys. Acta 491 (1977) 137-149. (doi: 10.1016/0005-2795(77)90049-6).

[S6] F.A. Walker, Magnetic, spectroscopic (EPR, ESEEM, Mössbauer, MCD and NMR) studies of low-spin ferriheme centers and their corresponding heme proteins, Coord. Chem Rev. 185-186 (1999) 471-513. (doi: 10.1016/S0010-8545(99)00029-6).

[S7] M.H.L. Pryce, Sign of g in magnetic resonance and the sign of the quadrupole moment of $\mathrm{Np}^{237}$, Phys. Rev. Lett. 3 (1959) 375-375. (doi: 10.1103/PhysRevLett.3.375).

[S8] A. Abragam, B. Bleaney, Electron Paramagnetic Resonance of Transitions Ions, Oxford University Press, Oxford, 1970. Chapter 3.

[S9] C.P. Poole, Jr. Electron Spin Resonance. A Comprehensive Treatise on Experimental Techniques. $2^{\text {nd }}$ edition. Wiley (N.Y.) 1983 (ISBN 0-471-04678-7). Ch.5, p189 and ff. 
